\title{
A FRACTAL-LIKE ALGEBRAIC SPIIITTING OF THE CLASSIFYING SPACE FOR VECTOR BUNDLES
}

\author{
V. GIAMBALVO, DAVID J. PENGELLEY AND DOUGLAS C. RAVENEL
}

\begin{abstract}
The connected covers of the classifying space $B O$ induce a decreasing filtration $\left\{B_{n}\right\}$ of $H_{*}(B O ; Z / 2)$ by sub-Hopf algebras over the Steenrod algebra $A$. We describe a multiplicative grading on $H_{*}(B O ; Z / 2)$ inducing a direct sum splitting of $B_{n}$ over $A_{n}$, where $\left\{A_{n}\right\}$ is the usual (increasing) filtration of $A$. The pieces in the splittings are finite, and the grading extends that of $H_{*} \Omega^{2} S^{3}$ which splits it into Brown-Gitler modules.

We also apply the grading to the Thomifications $\left\{M_{n}\right\}$ of $\left\{B_{n}\right\}$, where it induces splittings of the corresponding cobordism modules over the entire Steenrod algebra. These generalize algebraically the previously known topological splittings of the connective cobordism spectra $M O, M S O$ and MSpin.
\end{abstract}

Introduction. The classifying space for vector bundles, $B O$, is of longstanding interest in topology. We will describe a splitting of the mod 2 homology algebra of $B O$, having applications to connective cobordism Thom spectra. The splitting will be multiplicative; in other words it will be fully compatible with Whitney sums of vector bundles. It differs from other familiar splittings in topology in the way it interacts with the connected covers of $B O$ and the Steenrod algebra $A$ of cohomology operations. We will explain how this interaction is analogous to the geometric properties of the boundary of the fractal Mandelbrot set (or $M$-set) [PR].

The boundary of the $M$-set has two attributes: First, patterns become more elaborate upon magnification. Second, patterns visible at one level of magnification actually reappear under further magnification (self-similarity). The second property is Mandelbrot's idea of a fractal structure $[\mathbf{M}]$, while the first is an additional feature of certain fractals, like the boundary of the $M$-set. Our results about certain subalgebras of the algebra $H_{*} B O$ over the Hopf algebra $A$ reveal precisely these two features.

Specifically, consider the decreasing algebra filtration $\left\{B_{n}\right\}$ of $H_{*} B O$ provided by the images of the connected covers, and the standard increasing Hopf algebra filtration $\left\{A_{n}\right\}$ of $A$, where $A_{n}$ is generated by the first $2^{n}$ Steenrod squares. The analogy to the geometric properties of the boundary of the $M$-set is now made precise by interpreting "pattern" to mean a multiplicative direct sum splitting of an algebra $B_{n}$ over the Hopf algebra $A_{n}$, "magnification" as descending in the filtration $\left\{B_{n}\right\}$, and "more elaborate" as ascending in the filtration $\left\{A_{n}\right\}$.

Received by the editors May 15, 1987 .

1980 Mathematics Subject Classification (1985 Revision). Primary 55R40, 55R45, 57R90, 57T05.

Key words and phrases. Classifying space for vector bundles, connected covers of $B O$, connective cobordism Thom spectra, mod 2 homology algebra. 
Then the first property is that the multiplicative splitting we define on $H_{*} B O$ induces a direct sum splitting of each $B_{n}$ as an $A_{n}$-algebra. For instance, we obtain an $A_{0}$-algebra splitting of $H_{*} B O$, and an $A_{3}$-algebra splitting of $H_{*} B O\langle 8\rangle$. In [GPR] we showed how to build the various levels of magnification into a fractal Steenrod algebra preserving a multiplicative splitting of a fractal $H_{*} B O$.

The second (fractal-like) property is that patterns reappear after further magnification. We will show that this is also a characteristic of our filtration: The $A_{n-1}$-algebra $B_{n-1}$ (with its splitting as described above) reappears in rescaled form inside its own subalgebra $B_{n}$. In fact, we will show precisely how the $A_{n-1}$ algebra $B_{n}$ is built as a tensor product, with the main role played by many rescaled (i.e. redimensioned, but not merely suspended) copies of the $A_{n-1}$-algebra $B_{n-1}$.

Finally, since our splitting extends the well-known splitting [BP, Sn] of $H_{*} \Omega^{2} S^{3}$ into Brown-Gitler modules, and our summands are also finite, they could be regarded as a generalization of Brown-Gitler modules.

The two properties we have just informally described are illustrated by Figures 1 and 2, respectively. After we state the two main theorems precisely, the notation for the generators in the figures will be clear.

These results have immediate application to cobordism Thom spectra, in particular to the 7-connective cobordism spectrum $M O\langle 8\rangle$, an object of considerable interest for application in homotopy theory [BM, D1, D2, D3, DGIM, DM1, DM2, DM3]. We will use the fractal structure of $H_{*} B O$ to describe a ladderlike phenomenon, in which we see that each Thom subalgebra in the ladder of connected covers is built solely from copies of the connected cover from the next lower rung in the ladder, as follows: Let $M_{n}$ be the homology image in $H_{*} M O$ of the $n$th distinct connective cobordism spectrum (the Thomification of $B_{n}$ ). The coaction-quotient isomorphism $[\mathbf{K 1}, \mathbf{L i}, \mathbf{P 1}, \mathbf{P 2}]$ describes $M_{n}$ entirely in terms of the $A_{n-1}$ module structure of $B_{n}$ modulo a certain ideal $J_{n}$. Our splitting results will show that this $A_{n-1}$ algebra $B_{n} / J_{n}$ is essentially a product of many redimensioned copies of $B_{n-1}$. In short, $M_{n}$ can be completely described just using the next lower rung in the ladder of connected covers of $B O$. For instance, since $M_{3}$ is the homology of $M O\langle 8\rangle$, this latter result actually reduces $H_{*} M O\langle 8\rangle$ to understanding $H_{*} B S p i n$ as an $A_{2}$ module. Of course $H_{*} B S p i n$ is itself further split as an $A_{2}$ module by our results. This should lead to greater success in exploiting $M O\langle 8\rangle$ as a tool for understanding the stable homotopy groups of spheres.

A brief outline of our plan is as follows:

In $\S 1$ we state our main theorems precisely, indicate connections and applications to cobordism, and explain what we mean by a fractal $A$-splitting and a fractal $A$ map.

In $\S 2$ we set the stage for producing the fractal splittings by introducing the bipolynomial Hopf algebra generators over the two-local integers, describing how the connected cover images $B_{n}$ are generated by them, and how we will manage the action of Steenrod operations using nice two-local lifts provided by $T$. Lance.

In $\S 3$ we explain why and how we must modify the bipolynomial generators to see the fractal splitting. We give an explicit $Z_{(2)}$ formula for the modified generators, which hints at a relationship to Brown-Gitler spectra. We also discuss and record the properties they possess that will be crucial to demonstrating how they produce a fractal splitting. We defer the proof that they have these properties to a final section of the paper. 
GRADE 4 IN $H_{\star} B S O$ OVER $A_{1}$
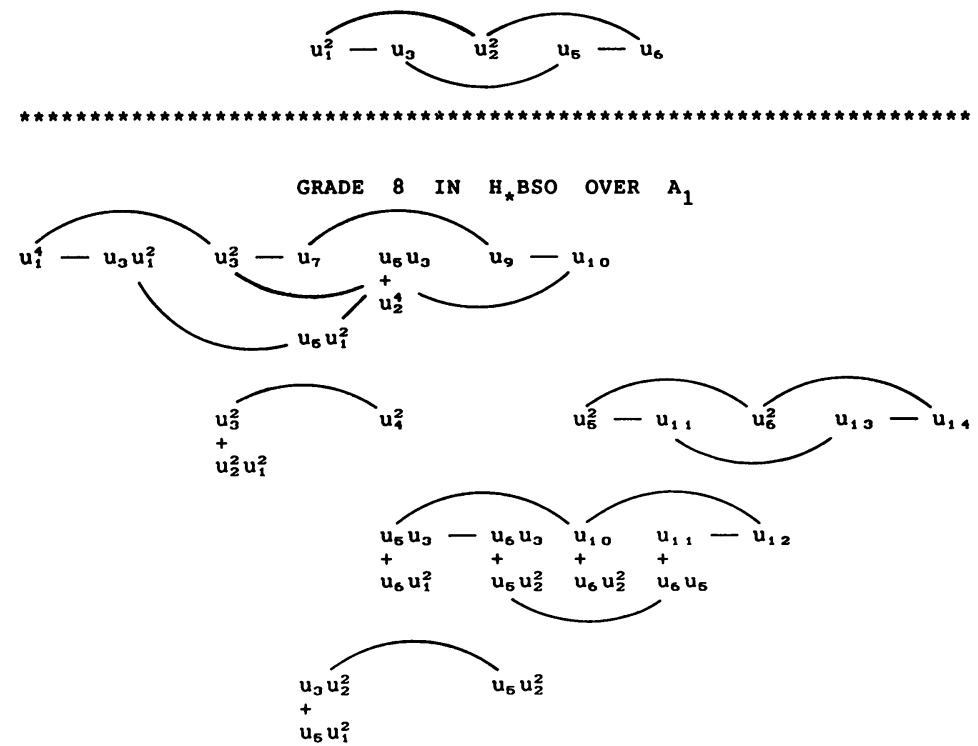

$u_{2}^{4}$

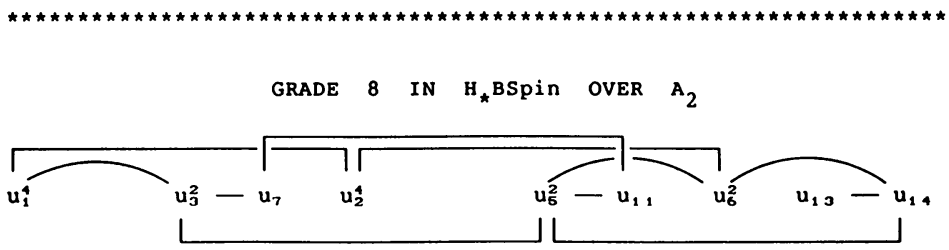

FIGURE 1. The splittings of Theorem 1.1 are illustrated by the first two grades in $H_{*} B S O=B_{1}$ over $A_{1}$, and the first grade in $H_{*} B S p i n=B_{2}$ over $A_{2}$

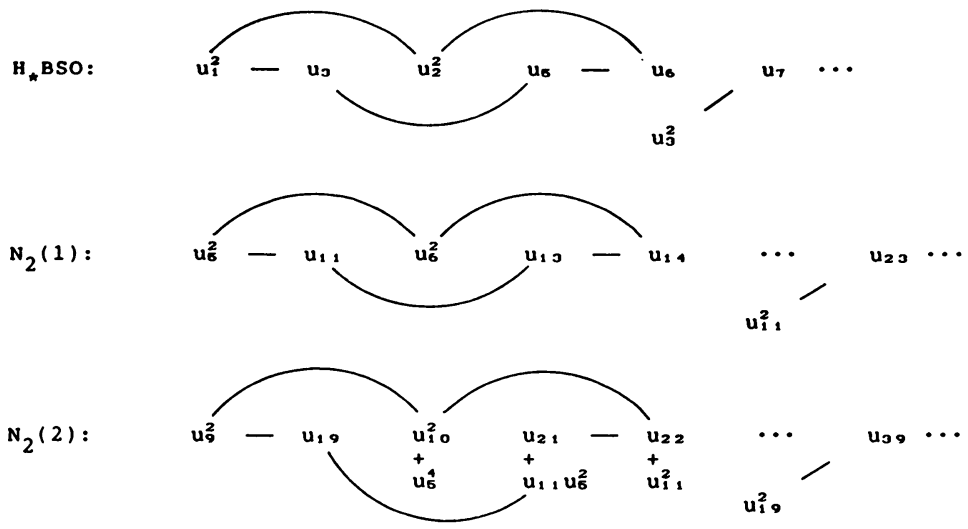

Figure 2. Theorem 1.2 is illustrated by the isomorphisms $f_{1}$ and $f_{2}$ carrying the $A_{1}$-algebra $H_{*} B S O=B_{1}$ to the tensor factors $N_{2}(1)$ and $N_{2}(2)$ of $H_{*} B S p i n=B_{2}$. Note that $f_{q}$ increases the dimension of a generator in grade $2^{m}$ by $2^{m+q}$. 
$\S 4$ is devoted solely to developing several $Z_{(2)}$ number-theoretic facts about binomial and multinomial coefficients, and about a certain formal power series, that will be needed later.

In $\S 5$ we prove our two main theorems using what we have previously assembled, and finish by showing that the fractal redimensionings in the second theorem actually do respect the grading of the splittings in the way one would hope.

$\S 6$ provides the proof we deferred in $\S 3$.

1. The splitting of $H_{*}(B O ; Z / 2)$, and its implications for cobordism. To state our main result on the algebraic splitting of $B O$, we need to establish a little notation regarding its connected covers. The $n$th distinct connected cover is $B O\langle\phi(n)\rangle$, where $\phi(n)$ is the dimension of the $n$th nontrivial homotopy group [St]. We will study its image

$$
B_{n}=\operatorname{Im}\left\{H_{*}(B O\langle\phi(n)\rangle ; Z / 2) \rightarrow H_{*}(B O ; Z / 2)\right\}
$$

in the homology of $B O$. For $n \leq 3$ this represents no loss, since the map in homology is injective for these first several connected covers, $B_{1}=H_{*} B S O, B_{2}=H_{*} B S p i n$, and $B_{3}=H_{*} B O\langle 8\rangle[\mathbf{S t}]$. For $n \geq 4$, however, they differ. $\tilde{H}_{*} B O\langle\phi(n)\rangle$ begins in dimension $\phi(n)$, which is approximately $2 n$, while $B_{n}$ begins in dimension $2^{n}$.

When reading Theorems 1.1 and 1.2, the reader should refer to Figures 1 and 2 in the Introduction, which provide low-dimensional illustrations of the structure the theorems provide.

Our first theorem will explicitly describe the Hopf subalgebras $B_{n}$ in a way that illustrates the fractal $A$-splitting over the Steenrod algebra. Let $\alpha(i)$ be the number of ones in the dyadic expansion of $i$.

THEOREM 1.1. There is a set of polynomial generators $\left\{u_{i}: i \geq 1\right\}$, with $u_{i}$ in dimension $i$, for $H_{*}(B O ; Z / 2)$, and an algebra grading \|\| defined on $H_{*}(B O ; Z / 2)$ by declaring $u_{i}$ to be homogeneous of grade $2^{m}$ where $2^{m}-1 \leq i<2^{m+1}-1$, such that:

(a) $B_{n}=Z / 2\left[u_{i}^{e(n, i)}: i \geq 1\right]$ where $e(n, i)=2^{\max \{0, n-(\alpha(i)-1)\}}$.

(b) For all $a \in A_{n}$ and all hornogeneous $b \in B_{n},\|(b) a\|=\|b\|$. Thus the grading induces a direct sum decomposition of $B_{n}$ over the subalgebra $A_{n}$ of the Steenrod algebra.

(c) The grading \|\| restricts to twice the familiar weight grading [BP] on

$$
H_{*}\left(\Omega^{2} S^{3}\right) \subset H_{*}(B O),
$$

since $u_{2^{m}-1}$ will be the coalgebra primitive in its dimension.

Some comments are in order about the theorem:

(1) The description in part (a) of polynomial generators for $B_{n}$ is much more transparent when one encapsulates it by noting that $u_{i}$ lies in $B_{\alpha(i)-1}$ but not in $B_{\alpha(i)}$, and that $\left(B_{n}\right)^{2} \subset B_{n+1}$.

(2) The obvious fact that the grading as defined on $H_{*} B O$ actually induces a grading on all the $B_{n}$ 's could be phrased by saying that the grading \|\| based on the $u_{i}$ 's is parallel to the filtration $\left\{B_{n}\right\}$.

(3) Part (b) is what we call a fractal action of the Steenrod algebra, where the splitting of the $B_{n}$ into finite summands according to the grading \|\| is respected by ever more of the Steenrod algebra as $n$ grows [GPR]. This is the fractal A-splitting 
that is analogous to the first geometric $M$-set property, namely more elaborate patterns under magnification.

This leads to the following useful terminology.

DEFINITION. An algebra homomorphism $f: H_{*}(B O ; Z / 2) \rightarrow H_{*}(B O ; Z / 2)$ is called a fractal $A$-map if $f \mid B_{n}$ is an $A_{n}$-module homomorphism.

Our second major theorem will utilize the generators $u_{i}$ from Theorem 1.1 to demonstrate the second (fractal) property for the filtration $\left\{B_{n}\right\}$, namely the rescaled reappearance of each $A_{n-1}$-algebra $B_{n-1}$ inside its own subalgebra $B_{n}$. This is the result which we will then apply to cobordism.

To state the theorem, we need some terminology regarding two particular subalgebras of $H_{*} B O$. One is

$$
L=Z / 2\left[u_{2^{m}-1}: m \geq 1\right]=H_{*}\left(\Omega^{2} S^{3}\right) \subset B_{0},
$$

the other is

$$
P=Z / 2\left[u_{2^{m}}: m \geq 1\right] \text {. }
$$

Recalling from 1.1(a) that the $u_{i}$ 's are parallel to $\left\{B_{n}\right\}$, we have the intersections

$$
L_{n}=L \cap B_{n}=Z / 2\left[u_{1}^{2^{n}}, u_{3}^{2^{n-1}}, \ldots, u_{2^{n+1}-1}, \ldots\right]
$$

and

$$
P_{n}=P \cap B_{n}=Z / 2\left[u_{2^{m}}^{2^{n}}: m \geq 1\right] .
$$

THEOREM 1.2. $H_{*} B O$ has a tensor product decomposition $L \otimes P \otimes\left[\bigotimes_{q \geq 1} N(q)\right]$ into polynomial subalgebras which satisfies:

(a) $N(q)$ has its polynomial generators precisely in dimensions $i+2^{m(i)+q}$ for all $i \geq 1$, where $m(i)=\left[\log _{2}(i+1)\right]$; in other words, $2^{m}-1 \leq i<2^{m+1}-1$, as in Theorem 1.1.

(b) Let $N_{n}(q)=N(q) \cap B_{n}$ for $n \geq 0$. Then the subalgebra $B_{n}$ actually decomposes into $L_{n} \otimes P_{n} \otimes\left[\otimes_{q \geq 1} N_{n}(q)\right]$ as a product of $A_{n-1}$-algebras.

(c) For every $q \geq 1$ there is an isomorphism $f_{q}: B_{0} \rightarrow N(q)$ of polynomial algebras, sending $u_{i}$ to a generator in dimension $i+2^{m(i)+q}$ (not necessarily $u_{i+2^{m(i)+q}}$ ), and its restriction induces an $A_{n-1}$-algebra isomorphism $f_{q}: B_{n-1} \rightarrow N_{n}(q)$ for every $n \geq 1$.

Two comments are in order about this theorem.

(1) Part (b) could be phrased by saying that the tensor product decomposition of $H_{*} B O$ is parallel to the filtration $\left\{B_{n}\right\}$, and fractal over $\left\{A_{n-1}\right\}$.

(2) Part (c) asserts the second (fractal) property, the rescaled reappearance of each $A_{n-1}$-algebra $B_{n-1}$ inside its subalgebra $B_{n}$, via fractal $A$-isomorphisms $f_{q}$.

The alert reader will realize that the mere existence of the fractal redimensioning isomorphisms $f_{q}$ of Theorem 1.2 ensures that a grading with the properties of Theorem 1.1 must exist, since $f_{q}$ increases the dimension of $u_{i}$ by $2^{q} \cdot 2^{m(i)}$, which is a constant times the underlying fractal grading. We will be using the bigrading provided by considering both dimension and fractal grading, and we can refer to elements as being homogeneous in either sense. In Theorem 1.2(c) we remarked that $f_{q}\left(u_{i}\right)$ is not necessarily $u_{i+2^{m(i)+q}}$. Nevertheless, we will prove in Lemma 5.13 that $f_{q}\left(u_{i}\right)$ is homogeneous with respect to the fractal grading as well as with respect to dimension. While we could in principle obtain Theorem 1.1 as a slick 
corollary to Theorem 1.2, this would undesirably obscure the nature of the grading, so we will provide a direct proof with explicit formulae for the $u_{i}$ 's.

Now we will provide an application of Theorem 1.2 to cobordism, to obtain a ladderlike decomposition of the $A$-algebras

$$
M_{n}=\operatorname{Im}\left\{H_{*}(M O\langle\phi(n)\rangle) \rightarrow H_{*}(M O)\right\},
$$

which correspond to the $B_{n}$ 's under the Thom isomorphism $H_{*}(M O) \cong H_{*}(B O)$. As before, $M_{n}$ actually coincides with the homology $H_{*}(M O\langle\phi(n)\rangle)$ of the cobordism Thom spectrum provided $n \leq 3$ (i.e. through $M O\langle 8\rangle$ ).

The analysis of $M_{n}$ is simplified by the coaction-quotient method [K1, Li, P1, P2], which provides $A$-algebra isomorphisms

$$
M_{n} \cong A^{*} \square_{A_{n-1}^{*}}\left(B_{n} / J_{n}\right) \cong\left(A^{*} \square_{A_{n-1}^{*}} Z / 2\right) \otimes\left(B_{n} / J_{n}\right),
$$

where $J$ is the ideal $\left(u_{1}, u_{3}, \ldots, u_{2^{m}-1}, \ldots\right)$ generated by the subalgebra $L=$ $H_{*}\left(\Omega^{2} S^{3}\right) \subset H_{*}(B O)$, and $J_{n}$ is the intersection ideal

$$
J \cap B_{n}=\left(u_{1}^{2^{n}}, u_{3}^{2^{n-1}}, \ldots, u_{2^{n+1}-1}, \ldots\right)
$$

generated by the subalgebra $L_{n}$. Note that $A$ denotes the Steenrod algebra, while $A^{*}$ is its dual.

Thus the main task in understanding the $A$-algebra $M_{n}$ is to describe the $A_{n-1^{-}}$algebra $B_{n} / J_{n}$. It is toward this goal that our application can aim, since Theorem 1.2 provides precisely an $A_{n-1}$-decomposition of $B_{n} / J_{n}$. So we have

COROLlaRY 1.3. $M_{n}$ is isomorphic as an A-algebra to

$$
A^{*} \square_{A_{n-1}^{*}}\left[P_{n} \otimes \bigotimes_{q \geq 1} N_{n}(q)\right]
$$

where $P_{n}$ is trivial over $A_{n-1}$, and every $N_{n}(q)$ is isomorphic as an $A_{n-1}$-algebra (up to redimensioning) to $B_{n-1}$.

COROLlaRY 1.4.

$$
H_{*}(M O\langle 8\rangle) \cong A^{*} \square_{A_{2}^{*}}\left[P_{3} \otimes \bigotimes_{q \geq 1}(\text { redimensioned }) H_{*}(\text { BSpin })\right]
$$

as A-algebras.

These corollaries provide the ladderlike description of the cobordism algebras that we alluded to in the Introduction. It was through empirical observation [GP] of these corollaries that we actually came to suspect the fractal structure for $H_{*} B O$ embodied in the two main theorems.

2. Lifting to the two-local bipolynomial generators. The main purpose of this section is to develop aspects of the bipolynomial Hopf algebra generators for $H_{*}(B O)$. Then we will be equipped to define the fractal generators $u_{i}$ which will enable us to prove the theorems of $\S 1$.

We will synthesize various features of the bipolynomial Hopf algebra generators $\left\{x_{i}\right\}$ developed by Husemoller [Hu], Baker [Ba], Kochman [K2], and Lance [La]. 
To do this, we must consider coefficients in $Z_{(2)}$, the integers localized at 2 , as well as $Z / 2$.

The doubling map [Sw, p. 494] gives an $A$-Hopf-algebra isomorphism

$$
H_{*}(B O ; Z / 2) \rightarrow H_{*}(B U ; Z / 2) .
$$

Let $\tilde{B}_{0}$ be $H_{*}\left(B U ; Z_{(2)}\right)$ with the dimensions of the generators halved. Then $\tilde{B}_{0}$ is a bipolynomial Hopf algebra [Ba], and $\tilde{B}_{0} \otimes Z / 2 \cong B_{0}=H_{*}(B O ; Z / 2)$. Let $d_{i} \in \tilde{B}_{0}$ be the coalgebra primitive in dimension $i$ dual to the Chern class $c_{i}$. A set of polynomial generators $\left\{x_{i}: i \geq 1\right\}$ for $\tilde{B}_{0}$ can be obtained from the primitives by the Witt polynomials

$$
d_{i}=d_{j \cdot 2^{k}}=\sum_{l=0}^{k} 2^{l} x_{j \cdot 2^{l}}^{2^{k-l}}
$$

where $i=j \cdot 2^{k}, j$ odd. Then $\tilde{B}_{0}=Z_{(2)}\left[x_{i}\right]$ and $H_{*}(B O ; Z / 2)=\tilde{B}_{0} \otimes Z / 2 \cong Z / 2\left[x_{i}\right]$. A simple proof that the $x_{i}$ 's are polynomial generators for $B_{0}$ can be found in [La]. Note that $x_{i}=d_{i}$ precisely when $i$ is odd. These generators are very well behaved with respect to the inclusions $B_{n} \subset B_{0}$. In fact the work of Baker [Ba] and Kochman [K2] implies the following

LEMMA 2.2. $B_{n}=Z / 2\left[x_{i}^{e(n, i)}: i \geq 1\right]$, where $e(n, i)=2^{\max \{0, n-(\alpha(i)-1)\}}$ as in Theorem 1.1.

To fully exploit this we need to describe the Steenrod algebra action on $H_{*}(B O)$ in terms of the $x_{i}$ 's. Lance [La] has constructed a $Z_{(2)}$ lift of the total dual square $\mathrm{Sq}=\sum_{t \geq 0} \mathrm{Sq}^{t}$. This lift, also denoted by Sq, satisfies the Cartan formula over $Z_{(2)}$ (Sq is a ring homomorphism) and has a particularly simple formula when evaluated on the primitives:

$$
d_{i} \mathrm{Sq}=\sum_{t \geq 0} \frac{i}{i-t}\left(\begin{array}{c}
i-t \\
t
\end{array}\right) d_{i-t}
$$

We will denote by $\gamma_{i, t}$ the coefficient

$$
\frac{i}{i-t}\left(\begin{array}{c}
i-t \\
t
\end{array}\right) \text {. }
$$

It is possible in theory to compute the action of the dual squares on the $x_{i}$ 's from this formula and (2.1), but the computations rapidly become intractible. Fortunately we can do most of the computations on the primitives. It is essential to observe that the nature of the Witt polynomials ensures that, to compute $x_{j .2^{k}}$ Sq mod 2, we need not know the $d_{j \cdot 2^{l}}$ Sq exactly for $l<k$, but only $\bmod 2^{l+1}$. The next lemma gives an example of how this idea will be applied.

LEMMA 2.4. Let $\tilde{f}: \tilde{B}_{0} \rightarrow \tilde{B}_{0}$ be an algebra map, and $f=\tilde{f} \otimes_{1} Z / 2$. Then $f\left(x_{j \cdot 2^{l}} \mathrm{Sq}^{t}\right)=f\left(x_{j \cdot 2^{l}}\right) \mathrm{Sq}^{t}$ for all $l \leq k, t \leq N$, if and only if $\tilde{f}\left(d_{j \cdot 2^{l}} \mathrm{Sq}^{t}\right) \equiv$ $\tilde{f}\left(d_{j \cdot 2^{l}}\right) \mathrm{Sq}^{t} \bmod 2^{l+1}$ for all $l \leq k, t \leq N$.

PROOF. Since we need to relate the polynomial generators to the primitives we must use the Witt polynomials (2.1). First note that the $Z_{(2)}$-Cartan formula 
gives the following congruence, which we will henceforth use frequently and without further comment. If $y \in \tilde{B}_{0}$, and $\tilde{f}\left(y \mathrm{Sq}^{t}\right) \equiv \tilde{f}(y) \mathrm{Sq}^{t} \bmod 2^{k}$ for all $t \leq N$, then $\tilde{f}\left(y^{2} \mathrm{Sq}^{t}\right) \equiv \tilde{f}\left(y^{2}\right) \mathrm{Sq}^{t} \bmod 2^{k+1}$ for all $t \leq N$. Now proceed by induction on $k$ as follows ( $k=0$ is not special). We will compare

$$
\tilde{f}\left(d_{j \cdot 2^{k}} \mathrm{Sq}^{t}\right)=\tilde{f}\left(2^{k} x_{j \cdot 2^{k}} \mathrm{Sq}^{t}\right)+\sum_{l=1}^{k} \tilde{f}\left(2^{k-l} x_{j \cdot 2^{k-l}}^{2^{l}} \mathrm{Sq}^{t}\right)
$$

with

$$
\tilde{f}\left(d_{j \cdot 2^{k}}\right) \mathrm{Sq}^{t}=\tilde{f}\left(2^{k} x_{j \cdot 2^{k}}\right) \mathrm{Sq}^{t}+\sum_{l=1}^{k} \tilde{f}\left(2^{k-l} x_{j \cdot 2^{k-l}}^{2^{l}}\right) \mathrm{Sq}^{t} .
$$

For either desired implication, the two summations are congruent $\bmod 2^{k+1}$ by induction and the above remark, so both implications are now immediate.

Many computations with the mod 2 Steenrod algebra are simplified by use of the Adem relations. While these do not lift, the integral two power squares on the integral primitives nevertheless do determine the entire mod 2 action. In particular

LEMMA 2.5. Let $f, \tilde{f}$ be as in Lemma 2.4. Then $f$ is a fractal $A$-map if and only if $\tilde{f}\left(d_{i}\right) \mathrm{Sq}^{2^{s}} \equiv \tilde{f}\left(d_{i} \mathrm{Sq}^{2^{s}}\right) \bmod 2^{\nu(i)+1}$ for all $s<\alpha(i)$.

PROOF. The forward implication is just a specialization from Lemma 2.4. In the other direction, to show that $f$ is a fractal $A$-map, it is clear from the mod 2 Adem relations and Lemma 2.2 that we need only show the mod 2 statement

$$
f\left(x_{i} \mathrm{Sq}^{2^{s}}\right)=f\left(x_{i}\right) \mathrm{Sq}^{2^{s}} \text { for } s<\alpha(i) .
$$

Proceeding by induction on $\nu(i)$, we consider the hypothesis

$$
\tilde{f}\left(d_{i} \mathrm{Sq}^{2^{s}}\right) \equiv \tilde{f}\left(d_{i}\right) \mathrm{Sq}^{2^{s}} \bmod 2^{\nu(i)+1} .
$$

Just as in the proof of (2.4), we consider the Witt sum for $d_{i}$ on each side, and note that by induction all but the first terms are pairwise congruent since, on all the bipolynomial generators in question, $\alpha$ is identical to $\alpha(i)$, but $\nu$ is less than $\nu(i)$. Thus the first pair matches also, i.e.

$$
\tilde{f}\left(2^{\nu(i)} x_{i} \mathrm{Sq}^{2^{s}}\right) \equiv \tilde{f}\left(2^{\nu(i)} x_{i}\right) \mathrm{Sq}^{2^{s}} \bmod 2^{\nu(i)+1}
$$

so we are done.

We mention in passing that the second author has developed an alternative approach to some of what follows using a lift of the total dual $\chi$ Sq. For the record we give its formula here:

$$
d_{i} \times \mathrm{Sq}=\sum_{t=0}^{i-1}(-1)^{t} \frac{i}{i+t}\left(\begin{array}{c}
i+t \\
t
\end{array}\right) d_{i-t} .
$$

This approach would eliminate the need for Lemma 4.7 and simplify the proof of Theorem 3.2(4). However, to use it here we would first need to develop its validity as a $Z_{(2)}$ lift, something Lance has already provided for the total dual Sq. 
3. The homogeneous splitting generators. Now that we have some information about $\tilde{B}_{0}$ we can define the polynomial generators $u_{i}$ which will induce the splitting we want to demonstrate. Given the nice description of the $B_{n}$ 's in terms of the $x_{i}$ 's, it would be fortunate if the $x_{i}$ 's themselves could simply be declared homogeneous, and our task completed. Perhaps the reader has already guessed, though, that things are not this easy. For instance, the relations $x_{2} \mathrm{Sq}^{1}=x_{1}$, $x_{3} \mathrm{Sq}^{1}=x_{1}^{2}, x_{4} \mathrm{Sq}^{1}=x_{3}+x_{2} x_{1}, x_{5} \mathrm{Sq}^{1}=x_{1}^{4}, x_{5} \mathrm{Sq}^{2}=x_{3}$ aptly illustrate the need both for corrections to the $x_{i}$ 's and for the use of $2^{m(i)}$ (with $m(i)=\left[\log _{2}(i+1)\right]$ ) to define the grading as a function of dimension of homogeneous generator. In particular, it seems we must correct $x_{5}$ to $u_{5}=x_{5}+x_{3} x_{1}^{2}$, so that $u_{5} \mathrm{Sq}^{2}=x_{3}$ and $u_{5} \mathrm{Sq}^{1}=0$, and thus the grading is preserved on $u_{5}$ by both $\mathrm{Sq}^{1}$ and $\mathrm{Sq}^{2}$, which is required since $\alpha(5)=2$.

We will actually "correct" the $x_{i}$ 's to produce our $u_{i}$ 's by providing explicit elements $\tilde{\tau}\left(d_{i}\right)$ to "correct" the integral primitives $d_{i}$. These corrections will in fact be chosen just from $\tilde{L}=Z_{(2)}\left[x_{2^{r}-1}: r \geq 1\right] \subset \tilde{B}_{0}$, as follows.

Let $l=\sum_{r \geq 1} x_{2^{r}-1}$, i.e. the formal sum of the generators in $\tilde{L}$. We begin by defining an algebra homomorphism $\tilde{\tau}: \tilde{B}_{0} \rightarrow \tilde{L}$ (which we think of as the correction to the $d_{i}$ 's) as follows:

$$
\tilde{\tau}\left(d_{i}\right)=\left.(-1)^{i} l^{2^{\bar{m}(i)+1}-i}\right|_{i}
$$

where $\bar{m}=\bar{m}(i)=\left[\log _{2}(i)\right]$, i.e. $\bar{m}$ is the integer satisfying $2^{\bar{m}} \leq i<2^{\bar{m}+1}$, and $\left.\right|_{i}$ means projection to dimension $i$. The reader should note the subtle (but crucial for our purposes) distinction between the function $\bar{m}(i)$ defined and used here and the function $m(i)=\left[\log _{2}(i+1)\right]$ in Theorems 1.1 and 1.2. They of course differ only when $i$ is one less than a power of 2 . In the future, if we write just $\bar{m}$ or $m$, the reader should carefully note its value from the context, particularly since it may be applied to various dimensions in the course of a single argument.

It is far from obvious that (3.1) even defines an algebra map as claimed, since the $d_{i}$ are only rational (not $Z_{(2)}$ ) algebra generators, and $\tilde{L}$ is only a $Z_{(2)}$-algebra. However, our next theorem will alleviate this concern.

Before continuing we pause to give an alternative formulation of (3.1) that hints at an explicit connection to Brown-Gitler spectra. Recall that (twice) the weight grading [BP] on $H_{*} \Omega^{2} S^{3}$ (which our grading will extend) is the algebra grading defined by $\left\|x_{2^{m}-1}\right\|=2^{m}$, and that it induces a splitting over the Steenrod algebra heralding the stable splitting of $\Omega^{2} S^{3}$ into Brown-Gitler spectra. Moreover, note that the grading extends naturally to $\tilde{L}$, and that the grade of a monomial equals its dimension plus its total exponent. This basic fact $($ grade $=\operatorname{dim}+\exp$ on $\tilde{L})$ enables one to rewrite (3.1) as

$$
\tilde{\tau}\left(d_{i}\right)=\left(\frac{1}{1+l}\right)_{\| 2^{\bar{m}(i)+1},\left.\right|_{i}},
$$

where the subscript $\|$ indicates projection to grade (not dimension) $2^{\bar{m}(i)+1}$. Moreover, in terms of $d=\sum_{i \geq 1} d_{i}$ we can go even further and write

$$
\tilde{\tau}(d)=\sum_{\bar{m} \geq 0}\left(\frac{1}{1+l}\right)_{\| 2^{\bar{m}+1}} ;
$$


in other words, $1 /(1+l)$ is followed by projection to all the Brown-Gitler modules of precisely two-power weight in $\tilde{L}$ (which do not, in fact, overlap in dimensions).

It is difficult for us to offer extensive further motivation for our formula for $\tilde{\tau}$. Suffice it to say that its crucial features will be "fractal compatibility" with the Steenrod algebra combined with $\tilde{\tau}\left(x_{2^{r}-1}\right)=-x_{2^{r}-1}$, the latter ensuring that $x_{2^{r}-1}$ will be "corrected" into oblivion. This key correction is necessary since the grading must be based on dimension via $m(i)=\left[\log _{2}(i+1)\right]$, not $\bar{m}(i)=\left[\log _{2}(i)\right]$. However, this "shift" to $m(i)$ from $\bar{m}(i)$ means that the Witt polynomials would no longer always show that homogeneous $x_{i}$ 's correspond to homogeneous $d_{i}$ 's, since the $x_{2^{r}-1}$ 's would have the wrong grade. But Lance's $Z_{(2)}$ squaring operation formulae make homogeneous $d_{i}$ 's appear most promising. Thus $\tilde{\tau}$ is designed to "homogenize" the $d_{i}$ 's by annihilating this obstruction without destroying the essence of Lance's formulae for the action of the integral squares. Much of the rest of the paper is devoted to showing that this vague strategy actually succeeds.

Our next step toward defining the $u_{i}$ 's is to define $\tilde{\rho}: \tilde{B}_{0} \rightarrow \tilde{B}_{0}$, the algebra map that will send $x_{i}$ to its "corrected" form $u_{i}$ (except when $i=2^{r}-1$, in which case we will make no correction). We want $\tilde{\rho}\left(d_{i}\right)=d_{i}+\tilde{\tau}\left(d_{i}\right)$, but merely defining $\tilde{\rho}=$ identity $+\tilde{\tau}$ will not produce an algebra map. We can, however, accomplish both purposes by defining $\tilde{\rho}$ to be the composition of algebra maps $\tilde{\mu}(1 \otimes \tilde{\tau}) \tilde{\Delta}$, where $\tilde{\Delta}$ is the diagonal, and $\tilde{\mu}$ the multiplication, in the Hopf algebra $\tilde{B}_{0}$. Finally we define $u_{i}$ by letting $u_{2^{r-1}}=x_{2^{r-1}}$ for all $r$, and $u_{i}=\tilde{\rho}\left(x_{i}\right)$ if $i \neq 2^{r}-1$.

We pause here to define important terminology for two qualitatively different types of Steenrod operations we will need to consider. Suppose $2^{m} \leq i \leq 2^{m+1}-$ 2. We will be considering operations $\mathrm{Sq}^{2^{s}}$ acting from dimension $i$ downward to dimension $i-2^{s}$, where $s<\alpha(i)$. If $i-2^{s} \geq 2^{m}$, in other words $i-2^{s}$ lies in the same range we specified for $i$, then we say the action of $\mathrm{Sq}^{2^{s}}$ on (dimension) $i$ is strict, because it remained between the same pair of two powers. On the other hand, if $i-2^{s}<2^{m}$, again with $s<\alpha(i)$, we say $\mathrm{Sq}^{2^{s}}$ is final on (dimension) $i$. We say this because a final operation can occur only in a very special way, as follows. Since $\alpha(i)>s$, and $2^{m} \leq i \leq 2^{m+1}-2$, clearly $i \geq 2^{m}+2^{s}-1$, i.e. $i-2^{s} \geq 2^{m}-1$. Thus the only way a final operation can occur is if it lands in precisely dimension $2^{m}-1$, and if $s$ actually equals $\alpha(i)-1$, i.e. $\mathrm{Sq}^{2^{s}}$ is the largest, or final, operation allowed (not every largest allowable operation is final, however).

The following theorem, establishing the crucial features of $\tau$ and $\tilde{\tau}$, will be proved in $\S 6$, after we develop some requisite $Z_{(2)}$ number theory.

Recalling from $\S 1$ that $L=\tilde{L} \otimes Z / 2$, we let $\tau=\tilde{\tau} \otimes Z / 2: B_{0} \rightarrow L$. As usual, denote by $\nu(i)$ the exponent of the largest two-power dividing $i$.

THEOREM 3.2. Let $\bar{m}=\bar{m}(i)$ as above. Then the homomorphisms $\tau$ and $\tilde{\tau}$ satisfy the following:

(1) $\tilde{\tau}$ extends uniquely to an algebra map $\tilde{B}_{0} \rightarrow \tilde{L}$.

(2) $\tilde{\tau}\left(x_{2^{r}-1}\right)=-x_{2^{r}-1}$ for all $r \geq 1$.

(3) $\tau\left(B_{n}\right) \subset B_{n}$.

(4) $\tilde{\tau}\left(d_{i}\right) \mathrm{Sq}^{2^{s}} \equiv \tilde{\tau}\left(d_{i} \mathrm{Sq}^{2^{s}}\right) \bmod 2^{\nu(i)+1}$ if $\mathrm{Sq}^{2^{s}}$ is strict on i, i.e. if $s<\alpha(i)$ and $2^{m} \leq i-2^{s}<i \leq 2^{m+1}-2$.

(5) $\tilde{\tau}\left(d_{i}\right) \mathrm{Sq}^{2^{s}} \equiv 0 \bmod 2^{\nu(i)+1}$ if $\mathrm{Sq}^{2^{s}}$ is final on $i$, i.e. if $s<\alpha(i)$ and $2^{m}-1=$ $i-2^{s}<i \leq 2^{m+1}-2$. 
The following corollary records resulting important properties of

$$
\rho=\tilde{\rho} \otimes Z / 2: B_{0} \rightarrow B_{0} .
$$

COROLLARY 3.3. (1) $\tilde{\rho}\left(x_{2^{r}-1}\right)=0$ for all $r \geq 1$.

(2) $\rho\left(x_{i}\right)$ is indecomposable if $i \neq 2^{r}-1$.

(3) $\rho\left(B_{n}\right) \subset B_{n}$.

(4) $\tilde{\rho}\left(d_{i}\right) \mathrm{Sq}^{2^{s}} \equiv \tilde{\rho}\left(d_{i} \mathrm{Sq}^{2^{s}}\right) \bmod 2^{\nu(i)+1}$ if $s<\alpha(i)$ and $\mathrm{Sq}^{2^{s}}$ is strict on $i$.

PROOF. Since $x_{2^{r-1}}=d_{2^{r-1}}$, and $\tilde{\rho} d_{i}=d_{i}+\tilde{\tau} d_{i}$, part (1) follows from (3.2)(2). The formula $\tilde{\rho}=\tilde{\mu}(1 \otimes \tilde{\tau}) \tilde{\Delta}$ shows that $\tilde{\rho}=$ identity $+\tilde{\tau} \bmod$ decomposables, and if $i \neq 2^{r}-1$, clearly, $\tilde{\tau} d_{i}$ is decomposable from (3.1). This proves (2).

Part (3) follows from $(3.2)(3)$ since $\rho$ is just the mod 2 reduction of $\tilde{\rho}=\tilde{\mu}(1 \otimes \tilde{\tau}) \tilde{\Delta}$, which preserves $B_{n}$ (recall $B_{n}$ is a Hopf-subalgebra, being the image of a space).

For part (4), we may use (3.2)(4) to compute

$$
\begin{aligned}
\tilde{\rho}\left(d_{i} \mathrm{Sq}^{2^{s}}\right) & =\gamma_{i, 2^{s}} \tilde{\rho}\left(d_{i-2^{s}}\right)=\gamma_{i, 2^{s}}\left(d_{i-2^{s}}+\tilde{\tau} d_{i-2^{s}}\right) \\
& =d_{i} \mathrm{Sq}^{2^{s}}+\tilde{\tau}\left(d_{i} \mathrm{Sq}^{2^{s}}\right) \equiv d_{i} \mathrm{Sq}^{2^{s}}+\left(\tilde{\tau} d_{i}\right) \mathrm{Sq}^{2^{s}} \\
& =\left(\tilde{\rho} d_{i}\right) \mathrm{Sq}^{2^{s}} \bmod 2^{\nu(i)+1} \cdot \square
\end{aligned}
$$

COROLlaRY 3.4. $B_{n}=Z / 2\left[u_{i}^{e(n, i)}: i \geq 1\right]$.

PROOF. If $i=2^{r}-1$ we have $u_{i}=x_{i}$. Otherwise $u_{i}=\rho\left(x_{i}\right) \equiv x_{i} \bmod$ decomposables by $(3.3)(2)$, and $u_{i} \in B_{\alpha(i)-1}$ by $(3.3)(3)$. Thus $u_{i}^{e(n, i)}$ is an indecomposable in $B_{n}$.

4. Some number theoretic lemmas. In this section we prove several disparate results of a purely number theoretic nature which we will need shortly.

The first lemma is a collection of facts, all of which are both well known and easily proved. We restate them here simply to allow the reader to follow the succeeding proofs more quickly. Proofs and/or references may be found in [Si]. Most date back to Legendre [Le].

LEMMA 4.1. For any positive integers $n, a, j$

(1) $\alpha(a+j) \leq \alpha(a)+\alpha(j)$.

(2) $\alpha(n+1)=\alpha(n)+1-\nu(n+1)$.

(3) If $a<2^{n}$ then $\alpha(a)+\nu(a) \leq n$.

(4) $\alpha\left(2^{n}-j\right)=n-\alpha(j)-\nu(j)+1$.

(5) $\nu\left(\begin{array}{c}2^{n} \\ j\end{array}\right)=n-\nu(j)$.

$$
\nu\left(\begin{array}{c}
a \\
r_{1}, r_{2}, \ldots, r_{s}
\end{array}\right)=\left[\sum \alpha\left(r_{i}\right)\right]-\alpha(a) .
$$

The next lemma is a special case of more general congruence results about binomial coefficients which will appear in [GMP].

LEMMA 4.2. Let $N$ and $s$ be positive integers. For any integer a (positive or negative!)

$$
\left(\begin{array}{c}
2^{N}+a \\
2^{s}-1
\end{array}\right) \equiv\left(\begin{array}{c}
a \\
2^{s}-1
\end{array}\right) \bmod 2^{s+1}
$$

if either $\nu(a+1) \leq N-s-1$ or $s \leq N-s$. 
PROOF. The binomial coefficient formula

$$
\left(\begin{array}{c}
x+y \\
z
\end{array}\right)=\sum_{t=0}^{z}\left(\begin{array}{l}
x \\
t
\end{array}\right) \cdot\left(\begin{array}{c}
y \\
z-t
\end{array}\right)
$$

obtained by expanding $(1+c)^{x+y}=(1+c)^{x}(1+c)^{y}$ yields

$$
\left(\begin{array}{c}
2^{N}+a \\
2^{s}-1
\end{array}\right)-\left(\begin{array}{c}
a \\
2^{s}-1
\end{array}\right)=\sum_{t=1}^{2^{s}-1}\left(\begin{array}{c}
2^{N} \\
t
\end{array}\right) \cdot\left(\begin{array}{c}
a \\
2^{s}-1-t
\end{array}\right)
$$

We will show that each term in the sum is divisible by $2^{s+1}$. Since $t \leq 2^{s}-1$ we must have $\nu(t) \leq s-1$. Now if $s \leq N-s$, we have

$$
\nu\left(\begin{array}{c}
2^{N} \\
t
\end{array}\right)=N-\nu(t) \geq N-s+1 \geq s+1 \text {. }
$$

On the other hand if $s>N-s$ and $\nu(a+1) \leq N-s-1$ we need to look at both factors. For the second factor we have

$\nu\left(\begin{array}{c}a \\ 2^{s}-1-t\end{array}\right)=\nu\left(\frac{2^{s}-t}{a+1}\left(\begin{array}{c}a+1 \\ 2^{s}-t\end{array}\right)\right) \geq \nu\left(2^{s}-t\right)-\nu(a+1) \geq \nu(t)-(N-s-1)$.

So

$$
\nu\left(\left(\begin{array}{c}
2^{N} \\
t
\end{array}\right) \cdot\left(\begin{array}{c}
a \\
2^{s}-1-t
\end{array}\right)\right) \geq N-\nu(t)+\nu(t)-N+s+1=s+1 .
$$

We will need two more specialized consequences of this:

COROllary 4.3. If $i<2^{N}$ and $s<\alpha(i)$ then

$$
(-1)^{i}\left(\begin{array}{c}
2^{s+1}+2^{N}-i \\
2^{s}
\end{array}\right) \frac{2^{N}-i}{2^{N}+2^{s+1}-i} \equiv(-1)^{i-2^{s}} \frac{i}{i-2^{s}}\left(\begin{array}{c}
i-2^{s} \\
2^{s}
\end{array}\right) \bmod 2^{\nu(i)+1} \text {. }
$$

PROOF. We begin by applying the previous lemma with $a=-2^{N}+i-2^{s}-1$. To do this we must first verify that either $s \leq N-s$ or $\nu(a+1) \leq N-s-1$. So suppose $s>N-s$. Then $i<2^{N}$ says $\alpha(i)+\nu(i) \leq N$, and $\alpha(i) \geq s+1$ gives $\nu(i) \leq N-s-1 \leq s-2$. Hence $\nu(a+1)=\nu\left(-2^{N}+i-2^{s}\right)=\nu(i) \leq N-s-1$. The lemma now gives

$$
\left(\begin{array}{c}
-2^{N}+i-2^{s}-1 \\
2^{s}-1
\end{array}\right) \equiv\left(\begin{array}{c}
i-2^{s}-1 \\
2^{s}-1
\end{array}\right) \bmod 2^{s+1}
$$

Using the identity

$$
\left(\begin{array}{l}
b \\
c
\end{array}\right)=(-1)^{c}\left(\begin{array}{c}
-b+c-1 \\
c
\end{array}\right)
$$

on the left side we can rewrite this as

$$
(-1)^{2^{s}-1}\left(\begin{array}{c}
2^{s+1}+2^{N}-i-1 \\
2^{s}-1
\end{array}\right) \equiv\left(\begin{array}{c}
i-2^{s}-1 \\
2^{s}-1
\end{array}\right) \bmod 2^{s+1}
$$

Now $2^{N} / 2^{s} \equiv 0 \bmod 2^{\nu(i)+1}$, since from above $\nu(i) \leq N-s-1$. With this information the reader may check that the congruence we seek now follows from (4.4). 
COROLlARY 4.5. If $i<2^{N}$ and $s<\alpha(i)$, then there is a congruence of Lance coefficients

$$
\gamma_{i, 2^{s}} \equiv \gamma_{i+2^{N}, 2^{s}} \bmod 2^{\nu(i)+1} .
$$

PROOF. This is similar in many ways to the previous corollary. We again apply Lemma 4.2 , this time to obtain

$$
\left(\begin{array}{c}
i-2^{s}-1 \\
2^{s}-1
\end{array}\right) \equiv\left(\begin{array}{c}
2^{N}+i-2^{s}-1 \\
2^{s}-1
\end{array}\right) \bmod 2^{s+1}
$$

by letting $a=i-2^{s}-1$. Our hypothesis again ensures that those of Lemma 4.2 are satisfied. Moreover as in the proof of $(4.3)$, we have $2^{N} / 2^{s} \equiv 0 \bmod 2^{\nu(i)+1}$, and thus the congruence above is equivalent to

$$
\frac{i}{i-2^{s}}\left(\begin{array}{c}
i-2^{s} \\
2^{s}
\end{array}\right) \equiv \frac{2^{N}+i}{2^{N}+i-2^{s}}\left(\begin{array}{c}
2^{N}+i-2^{s} \\
2^{s}
\end{array}\right) \quad \bmod 2^{\nu(i)+1},
$$

which is the desired congruence among Lance coefficients.

When we prove Theorem 3.2 we will need

LEMMA 4.6. The multinomial coefficient

$$
\left(\begin{array}{c}
2^{N}-i \\
r_{1}, r_{2}, \ldots, r_{s}
\end{array}\right) \equiv 0 \bmod 2^{\nu(i)+1}
$$

if $\sum_{t=1}^{s} 2^{t} r_{t}=2^{N}, i<2^{N}$, and $\nu\left(r_{l}\right)<\alpha(i)-l$ for some $l$.

PrOOF. Let $i=j \cdot 2^{k}, j$ odd. Then we need to show under the above conditions that

$$
\nu\left(\begin{array}{c}
2^{N}-i \\
r_{1}, r_{2}, \ldots, r_{s}
\end{array}\right) \geq k+1
$$

Now

$$
\begin{aligned}
\nu\left(\begin{array}{c}
2^{N}-i \\
r_{1}, r_{2}, \ldots, r_{s}
\end{array}\right) & =\sum \alpha\left(r_{t}\right)-\alpha\left(2^{N}-i\right) \\
& =\sum \alpha\left(r_{t}\right)-\alpha\left(2^{N-k}-j\right)=\sum \alpha\left(r_{t}\right)-(N-k+1-\alpha(j)) \\
& =\sum_{t \neq l} \alpha\left(r_{t}\right)-N+k-1+\alpha(j)+\alpha\left(r_{l}\right) \\
& \geq \sum_{t \neq l} \alpha\left(r_{t}\right)-N+k+l+\nu\left(r_{l}\right)+\alpha\left(r_{l}\right) \\
& =\sum_{t \neq l} \alpha\left(r_{t}\right)-N+k+l+\alpha\left(r_{l}-1\right)+1 \\
& =\sum_{t \neq l} \alpha\left(2^{t} r_{t}\right)+\alpha\left(2^{l} r_{l}-2^{l}\right)-N+k+l+1 \\
& \geq \alpha\left(2^{N}-2^{l}\right)-N+k+l+1 \\
& =N-l-N+k+l+1=k+1 .
\end{aligned}
$$

Our final lemma will allow us to compute with an alternate $Z_{(2)}$ lift of the Steenrod squares that we will define in $\S 6$. 
LEMMA 4.7. Let $f(x)$ be the formal power series

$$
\sum_{t \geq 0}\left(\begin{array}{c}
2 t+1 \\
t
\end{array}\right) \frac{1}{2 t+1} x^{t+1}
$$

Then for all positive $q$,

$$
(f(x))^{q}=\sum_{t \geq 0}\left(\begin{array}{c}
2 t+q \\
t
\end{array}\right) \frac{q}{2 t+q} x^{t+q} .
$$

PROOF. The series $f(x)$ is familiar to combinatorists, the coefficients being the Catalan numbers. Many properties of the series are well known, including that it satisfies the quadratic equation $(f(x))^{2}=f(x)-x$ [Br, p. 143f]. Thus we have $(f(x))^{q}=(f(x))^{q-1}-x(f(x))^{q-2}$. Inductively we have

$$
(f(x))^{q}=\sum_{t \geq 0}\left(\left(\begin{array}{c}
2 t+q-1 \\
t
\end{array}\right) \frac{q-1}{2 t+q-1}-\left(\begin{array}{c}
2 t+q-2 \\
t
\end{array}\right) \frac{q-2}{2 t+q-2}\right) x^{t+q-1} .
$$

Letting $v=t-1$, the coefficient of $x^{q+v}$ is

$$
\begin{aligned}
& \left(\begin{array}{c}
2 v+q+1 \\
v+1
\end{array}\right) \frac{q-1}{2 v+q+1}-\left(\begin{array}{c}
2 v+q \\
v+1
\end{array}\right) \cdot\left(\begin{array}{c}
q-2 \\
2 v+2
\end{array}\right) \\
& \quad=\left(\begin{array}{c}
2 v+q \\
v
\end{array}\right) \cdot\left[\frac{q-1}{v+1}-\frac{(v+q)(q-2)}{(v+1)(2 v+q)}\right]=\left(\begin{array}{c}
2 v+q \\
v
\end{array}\right) \frac{q}{2 v+q} .
\end{aligned}
$$

5. Proofs of the main theorems. In this section the proofs of Theorems 1.1 and 1.2 are completed. First consider Theorem 1.1, restated here for convenience.

THEOREM 1.1. There is a set of polynomial generators $\left\{u_{i}: i \geq 1\right\}$, with $u_{i}$ in dimension $i$, for $H_{*}(B O ; Z / 2)$, and an algebra grading \|\| defined on $H_{*}(B O ; Z / 2)$ by declaring $u_{i}$ to be homogeneous of grade $2^{m}$ where $2^{m}-1 \leq i<2^{m+1}-1$, such that:

(a) $B_{n}=Z / 2\left[u_{i}^{e(n, i)}: i \geq 1\right]$ where $e(n, i)=2^{\max \{0, n-(\alpha(i)-1)\}}$.

(b) For all $a \in A_{n}$ and all homogeneous $b \in B_{n},\|(b) a\|=\|b\|$. Thus the grading induces a direct sum decomposition of $B_{n}$ over the subalgebra $A_{n}$ of the Steenrod algebra.

(c) The grading \|\| restricts to twice the familiar weight grading $[\mathbf{B P}]$ on

$$
H_{*}\left(\Omega^{2} S^{3}\right) \subset H_{*}(B O),
$$

since $u_{2^{m}-1}$ will be the coalgebra primitive in its dimension.

PROOF. In $\S 2$ we defined $u_{i}$ as

$$
u_{i}= \begin{cases}\tilde{\rho}\left(x_{i}\right) & \text { for } i \neq 2^{r}-1 \\ x_{2^{r}-1} & \text { for } i=2^{r}-1\end{cases}
$$

and Corollary 3.4 gives part (a) of the theorem.

We define a grading on $\tilde{B}_{0}$ by

$$
\left\|u_{i}\right\|=2^{m}
$$

where $m=m(i)=\left[\log _{2}(i+1)\right]$, i.e., $2^{m}-1 \leq i<2^{m+1}-1$, as usual. Since $u_{2^{r-1}}=x_{2^{r-1}}$, and $H_{*} \Omega^{2} S^{3} \subset B_{0}$ is $Z / 2\left[x_{2^{r-1}}\right]$, we have part (c) of the theorem. 
To prove part (b) we need to show that $A_{n}$ respects the grading on $B_{n}$, i.e., for all $a \in A_{n}$ and homogeneous $b \in B_{n},\|(b) a\|=\|b\|$. From the Cartan formula and Corollary 3.4 we see that it is sufficient to show that

$$
\left\|u_{i} \mathrm{Sq}^{2^{s}}\right\|=\left\|u_{i}\right\| \text { for } s<\alpha(i)
$$

To do this we need to go back to $\tilde{B}_{0}=Z_{(2)}\left[u_{i}\right]$. Since we are abusing notation by considering $u_{i}$ both in $B_{0}$ and $\tilde{B}_{0}$, we will be careful to make statements "mod 2 " if this is all we claim. Our present goal is to prove (5.2) mod 2.

It is worthwhile at this point to consider again why this may work. First, simple facts like $x_{2} \mathrm{Sq}^{1}=x_{1}$ mod 2 show that one must define the grading using $m(i)$ (shifted two-power blocks) rather than $\bar{m}(i)$ as in (3.1). On the other hand, if we were to define the grading using $m(i)$ as in (5.1), but making the generators $x_{i}$ homogeneous (rather than the $u_{i}$ ), then the primitives

$$
d_{j \cdot 2^{k}}=\sum_{l=0}^{k} 2^{l} x_{j \cdot 2^{l}}^{2^{k-l}}
$$

would not be homogeneous (even though Lance's Steenrod squares (2.3) suggest trying to do this) if $k$ is positive and $j$ is one less than a power of 2 . For example, consider $d_{12}=4 x_{12}+2 x_{6}^{2}+x_{3}^{4}$. Then $x_{12}$ and $x_{6}^{2}$ would have grade 8 , but $x_{3}^{4}$ would have grade 16 . The map $\rho$ is designed to remedy these incompatibilities. In particular, if $j=2^{r}-1$, then $\tilde{\rho}\left(x_{j}\right)=0$ from $(3.3)(1)$, so $\tilde{\rho} d_{j \cdot 2^{k}}=\sum_{l=1}^{k} 2^{l} u_{j \cdot 2^{l}}^{2^{k-l}}$ (note $l$ now starts at 1 ), and this $i s$ homogeneous. Now, according to our guiding principle, as long as the appropriate Steenrod operations preserve homogeneity on the $\tilde{\rho}\left(d_{i}\right)$ 's (and the $u_{2^{r}-1}$ 's), we can "solve" for the $u_{i}$ 's, which are just $\rho\left(x_{i}\right)$ 's, and retain homogeneity under the fractal $A$-action. We now proceed to prove (5.2).

First consider $i=2^{r}-1$, recalling that $u_{2^{r-1}}=x_{2^{r-1}}=d_{2^{r}-1}$. Then (5.2) holds, since the Lance coefficients in (2.3) yield $u_{2^{r}-1} \mathrm{Sq}^{1} \equiv u_{2^{r-1}-1}^{2} \bmod 2$ and $u_{2^{r-1}} \mathrm{Sq}^{2^{s}} \equiv 0 \bmod 2$ for $s>0$.

Now for $i \neq 2^{r}-1$, let $i=j \cdot 2^{k}, j$ odd, and $m=\left[\log _{2}(i+1)\right]$ as usual, and proceed by induction on $i$. We have

$$
\tilde{\rho} d_{i}=\tilde{\rho} d_{j \cdot 2^{k}}=2^{k} u_{j \cdot 2^{k}}+\sum_{l=b}^{k-1} 2^{l} u_{j \cdot 2^{l}}^{2^{k-l}}
$$

where $b=1$ if $j=2^{r}-1$ and $b=0$ otherwise.

Note that $\alpha\left(j \cdot 2^{l}\right)=\alpha(j)=\alpha(i)$ for all $l$, so the values of $s$ relevant in (5.2) are identical for all the $u$ 's in (5.3). Therefore from the induction assumption, the $\bmod 2$ Adem relations, the Cartan formula, and the fact that $j \cdot 2^{l} \neq 2^{r}-1$, we have

$$
u_{j \cdot 2^{l}} \mathrm{Sq}^{t} \quad \text { is homogeneous of grade } 2^{m-k} \cdot 2^{l} \bmod 2
$$

for $l<k, t \leq 2^{s}$. Thus so is $\sum_{t=0}^{2^{s}} u_{j \cdot 2^{l}} \mathrm{Sq}^{t}$, and hence $\left(\sum_{t=0}^{2^{s}} u_{j \cdot 2^{l}} \mathrm{Sq}^{t}\right)^{2^{k-l}}$ is homogencous of grade $2^{m} \bmod 2^{k+1-l}$. Thus the $Z_{(2)}$ Cartan formula ensures that

$$
u_{j \cdot 2^{l}}^{2^{k-1}} \mathrm{Sq}^{2^{s}} \text { is homogeneous of grade } 2^{m-l} \bmod 2^{k+1} .
$$


Now applying $\mathrm{Sq}^{2^{s}}$ to (5.3) we get

$$
\tilde{\rho}\left(d_{i}\right) \mathrm{Sq}^{2^{s}}=2^{k} u_{j \cdot 2^{k}} \mathrm{Sq}^{2^{s}}+\sum_{l=b}^{k-1} 2^{l}\left(u_{j \cdot 2^{l}}^{2^{k-l}}\right) \mathrm{Sq}^{2^{s}}
$$

From above we know the summation is homogeneous of grade $2^{m} \bmod 2^{k+1}$, and we will show that the left-hand side enjoys the same feature. There are two cases:

If the operation is strict, then by $(3.3)(4)$ we have

$$
\tilde{\rho}\left(d_{i}\right) \mathrm{Sq}^{2^{s}} \equiv \tilde{\rho}\left(d_{i} \mathrm{Sq}^{2^{s}}\right)=\gamma_{i, 2^{s}} \tilde{\rho}\left(d_{i-2^{s}}\right) \bmod 2^{k+1},
$$

and the latter is homogeneous of grade $2^{m} \bmod 2^{k+1}$ since in this case $m\left(i-2^{s}\right)=$ $m(i)=m$.

On the other hand, if the operation is final, then

$$
\begin{aligned}
\tilde{\rho}\left(d_{i}\right) \mathrm{Sq}^{2^{s}} & =d_{i} \mathrm{Sq}^{2^{s}}+\tilde{\tau}\left(d_{i}\right) \mathrm{Sq}^{2^{s}} \equiv \gamma_{i, 2^{s}} d_{2^{m}-1}+0 \\
& =\gamma_{i, 2} u_{2^{m}-1} \quad \bmod 2^{k+1}
\end{aligned}
$$

by $(3.2)(5)$ and our discussion when we defined final operations. Of course $u_{2^{m}}-1$ is homogeneous of grade $2^{m}$ by definition.

So $2^{k} u_{j \cdot 2^{k}} \mathrm{Sq}^{2^{s}}$ must also be homogeneous of grade $2^{m} \bmod 2^{k+1}$, and therefore $u_{j \cdot 2^{k}} \mathrm{Sq}^{2^{3}}$ is homogeneous of grade $2^{m} \bmod 2$. This completes the proof of Theorem 1.1 .

We now turn our attention to the proof of

THEOREM 1.2. $H_{*} B O$ has a tensor product decomposition $L \otimes P \otimes\left[\bigotimes_{q \geq 1} N(q)\right]$ into polynomial subalgebras which satisfies:

(a) $N(q)$ has its polynomial generators precisely in dimensions $i+2^{m(i)+q}$ for all $i \geq 1$, where $m(i)=\left[\log _{2}(i+1)\right]$; in other words, $2^{m}-1 \leq i<2^{m+1}-1$, as in Theorem 1.1.

(b) Let $N_{n}(q)=N(q) \cap B_{n}$ for $n \geq 0$. Then the subalgebra $B_{n}$ actually decomposes into $L_{n} \otimes P_{n} \otimes\left[\otimes_{q \geq 1} N_{n}(q)\right]$ as a product of $A_{n-1}$-algebras.

(c) For every $q \geq 1$ there is an isomorphism $f_{q}: B_{0} \rightarrow N(q)$ of polynomial algebras, sending $u_{i}$ to a generator in dimension $i+2^{m(i)+q}$ (not necessarily $u_{i+2^{m(i)+q}}$ ), and its restriction induces an $A_{n-1}$-algebra isomorphism $f_{q}: B_{n-1} \rightarrow N_{n}(q)$ for every $n \geq 1$.

To construct the decomposition we explicitly construct the isomorphisms involved, as follows. ing:

LEMMA 5.4. There are fractal $A$-algebra maps $f_{q}: B_{0} \rightarrow B_{0}$ for $q \geq 1$ satisfy-

(1) Each $f_{q}\left(u_{i}\right)$ is an indecomposable in dimension $i+2^{m(i)+q}$, and thus $f_{q}$ is a monomorphism.

(2) $f_{q}\left(B_{n}\right) \subset B_{n+1}$.

PROOF. In order to define $f_{q}$, we need a map that looks very similar, but is in fact quite different. The idea is to try to send $u_{i}$ to $u_{i+2^{m+q}}$, but this will not work if $q>1$. We need to correct the generators in the image, just as we corrected the 
$x_{i}$ 's to get $u_{i}$ 's. As usual, $m=m(i)$ is given by $2^{m}-1 \leq i \leq 2^{m+1}-2$. Define $\tilde{g}_{q}: \tilde{B}_{0} \rightarrow \tilde{B}_{0}$ by

$$
\begin{aligned}
& \tilde{g}_{q}\left(x_{i}\right)=\left\{\begin{array}{ll}
u_{i+2^{m+q}} & \text { for } i \neq 2^{r}-1 \\
u_{i+2^{m+q}-1} & \text { for } i=2^{r}-1
\end{array} \text { if } q>1,\right. \\
& \tilde{g}_{1}\left(x_{i}\right)=0
\end{aligned}
$$

on the generators $x_{i}$ and extend as an algebra map. Then define

$$
\tilde{f}_{q}\left(u_{i}\right)=u_{i+2^{m+q}}+\tilde{g}_{q}\left(u_{i}-x_{i}\right)
$$

This can be written as

$$
\tilde{f}_{q}\left(u_{i}\right)= \begin{cases}u_{i+2^{m+q}} & \text { for } i=2^{r}-1 \text { or } q=1, \\ \tilde{g}_{q}\left(u_{i}\right) & \text { otherwise. }\end{cases}
$$

Let $g_{q}=\tilde{g}_{q} \otimes Z / 2$ and $f_{q}=\tilde{f}_{q} \otimes Z / 2$ be the $\bmod 2$ reductions, as usual. (Note that for $q>1 g_{q}\left(x_{2^{r}-1}\right)$ is very different from $f_{q}\left(x_{2^{r}-1}\right)$ since $g_{q}$ uses $\bar{m}$, while $f_{q}$ uses $m$, in determining the two-power added to the dimension.)

Since $x_{i}, u_{i} \in B_{\alpha(i)-1}$ for all $i,(5.5)$ shows that $g_{q}\left(B_{n}\right) \subset B_{n+1}$, and thus the same is true of $f_{q}$, so (2) holds.

Now we will work to show that each $f_{q}$ is a fractal $A$-map. To do this we will show

$$
\tilde{f}_{q}\left(\tilde{\rho} d_{i}\right) \mathrm{Sq}^{2^{s}} \equiv \tilde{f}_{q}\left(\left(\tilde{\rho} d_{i}\right) \mathrm{Sq}^{2^{s}}\right) \bmod 2^{\nu(i)+1}
$$

for $s<\alpha(i), i \neq 2^{r}-1$, and then apply Lemma 2.5 , or rather a slight variation of it, as we now explain. The point is that we wish to replace $d_{i}$ by $\tilde{\rho} d_{i}$ in Lemma 2.5 . This is no great problem, since $\tilde{\rho} d_{j \cdot 2^{k}}$ with $j$ odd is also built via Witt sums from the $\tilde{\rho} x_{j \cdot 2}$ 's, which play the same role in the $B_{n}$ 's as the $x_{j \cdot 2}$ 's , with the exception of the $x_{2^{r}-1}$ 's (recall $\tilde{\rho} x_{2^{r}-1}=0$ ). Therefore, all we need to do, in addition to proving (5.8), is show that $f_{q}$ commutes with the appropriate (i.e. fractal) Steenrod operations on the generators $x_{2^{r-1}}=u_{2^{r-1}}$. We will do this straightforward mod 2 computation first. We have, for all $q$,

$$
f_{q}\left(x_{2^{r}-1} \mathrm{Sq}^{2^{s}}\right)=f_{q}\left(\delta_{0, s} x_{x^{r-1}-1}^{2}\right)=\delta_{0, s} u_{2^{r+q-1}+2^{r-1}-1}^{2},
$$

with the Kronecker $\delta_{0, s}$ nonzero only for $s=0$. On the other hand, we can compute

$$
\left(f_{q} x_{2^{r-1}}\right) \mathrm{Sq}^{2^{s}}=u_{2^{r+a}+2^{r-1}} \mathrm{Sq}^{2^{s}}=\rho\left(x_{2^{r+a}+2^{r-1}}\right) \mathrm{Sq}^{2^{s}}=\rho\left(d_{2^{r+q}+2^{r}-1}\right) \mathrm{Sq}^{2^{s}} \text {. }
$$

To the latter we may apply (3.3)(4) (since $s \leq r-1$ implies $\left(2^{r+q}+2^{r}-1\right)-2^{s} \geq$ $2^{r+q}$, i.e. the operation is strict), so it equals

$$
\gamma_{2^{r+q}+2^{r}-1,2^{s}} \rho\left(d_{2^{r+q}+2^{r}-2^{s}-1}\right)=\delta_{0, s} u_{2^{r+q-1}+2^{r-1}-1}^{2} \bmod 2,
$$

as claimed, since $s \leq r-1$ ensures that the Lance coefficient is $\delta_{0, s} \bmod 2$.

Now we turn to proving (5.8). If $i=2^{r}-1$, both sides are zero. If, however, $i \neq 2^{r}-1$, we first claim that

$$
\tilde{f}_{q}\left(\tilde{\rho} d_{i}\right)=\tilde{\rho}\left(d_{i+2^{m+q}}\right)+\tilde{g}_{q}\left(\tilde{\rho}\left(d_{i}\right)-d_{i}\right)=\tilde{\rho}\left(d_{i+2^{m+q}}\right)+\tilde{g}_{q}\left(\tilde{\tau} d_{i}\right)
$$

where $2^{m} \leq i<2^{m+1}-1$.

The subtlety of this claim is illustrated by the fact that it is clearly false for $i=2^{r}-1$, but we assert it nevertheless holds for all other differences of two twopowers. We will sketch the four cases to consider: 
First, if $i$ is not a difference of two two-powers, then neither is $i+2^{m+q}$. So a Witt sum may be formed using (5.7) to obtain either $\tilde{f}_{1} \tilde{\rho} d_{i}=\tilde{\rho} d_{i+2^{m+1}}$ as desired, if $q=1$, or if $q>1, \tilde{f}_{q} \tilde{\rho} d_{i}=\tilde{g}_{q} \tilde{\rho} d_{i}=\tilde{g}_{q}\left(d_{i}+\tilde{\tau} d_{i}\right)$, which in turn by (5.5) is $\tilde{\rho} d_{i+2^{m+q}}+\tilde{g}_{q} \tilde{\tau} d_{i}$.

On the other hand, if $i$ is a difference of two two-powers, and $q=1$, but $i \neq 2^{r}-1$, then $i+2^{m+1}$ is also a difference of two two-powers, but not one less than a power of 2 , and one can check that both $\tilde{\rho} d_{i}$ and $\tilde{\rho} d_{i+2^{m+1}}$ are Witt sums with a term missing at the bottom, so by (5.7) $\tilde{f}_{1}$ carries the one sum to the other, as desired (this fails for $i=2^{r}-1$ because of the difference between $m$ and $\bar{m}$ ). Finally, if $i$ is a difference of two two-powers, but $q>1$ and $i \neq 2^{r}-1$, then $i+2^{m+q}$ is not a difference of two two-powers, and we proceed rather carefully, as follows. In this case, clearly $\tilde{f}_{q}\left(\tilde{\rho} d_{i}\right)=\tilde{g}_{q}\left(\tilde{\rho} d_{i}\right)$, so we only need show that $\tilde{\rho}\left(d_{i+2^{m+q}}\right)-\tilde{g}_{q} d_{i}=0$. This follows from the definition of $\tilde{g}_{q}$ in (5.5), taking special note that the two bottom terms in the Witt sums both in effect use $\bar{m}(j)$ rather than $m(j)$, and hence match. (This too would fail if $i=2^{r}-1$, due to the difference between $\bar{m}(i)$ and $m(i)$.)

We now prove (5.8) by using formula (5.9). As a first step we show that

$$
\tilde{g}_{q}\left(\tilde{\tau}\left(d_{i}\right)\right) \mathrm{Sq}^{2^{s}} \equiv \tilde{g}_{q}\left(\tilde{\tau}\left(d_{i}\right) \mathrm{Sq}^{2^{s}}\right) \bmod 2^{k+1}
$$

for $s<\alpha(i)$.

To prove this, first note that $g_{q}$ is a fractal $A$-map when restricted to $L$, i.e.

$$
g_{q}\left(x_{2^{r}-1}\right) \mathrm{Sq}^{2^{s}}=g_{q}\left(x_{2^{r}-1} \mathrm{Sq}^{2^{s}}\right) \text { for } s<r .
$$

To see this, observe that if $q=1, g_{1}\left(x_{2^{r}-1}\right)=0$, so $g_{1}$ commutes with all Steenrod operations, while if $q>1, g_{q}\left(x_{2^{r}-1}\right)=u_{2^{r}-1+2^{q+r-1}}$, and the calculation is now similar to the mod 2 calculation we already made earlier in the proof for $f_{q}$ on $x_{2^{r}-1}$ (the requisite application of $(3.3)(4)$ requires $q>1$ though, in this case). Now since $\tau\left(B_{n}\right) \subset L \cap B_{n}=L_{n}$ by $(3.2)(3), \tilde{\tau}\left(d_{i}\right)$ is actually a Witt polynomial in $L_{n}$ (not just in $L) \bmod 2^{k+1}$, so $(5.10)$ holds since $g_{q}$ is fractal on $L$.

From this point on we must consider strict and final operations separately:

Case I. $\mathrm{Sq}^{2}$ is strict on dimension $i$.

With $\mathrm{Sq}^{2^{s}}$ strict on $i$, we can take (5.10) one step further using (3.2)(4) to obtain

$$
g_{q}\left(\tilde{\tau}\left(d_{i}\right)\right) \mathrm{Sq}^{2^{s}} \equiv \tilde{g}_{q}\left(\tilde{\tau}\left(d_{i} \mathrm{Sq}^{2^{s}}\right)\right) \bmod 2^{k+1} .
$$

Now we are ready to complete the verification of (5.8) in this case. Using (5.9) and (5.11) the left side of $(5.8)$ is

$$
\begin{aligned}
\tilde{f}_{q}\left(\tilde{\rho} d_{i}\right) \mathrm{Sq}^{2^{s}} & =\tilde{\rho}\left(d_{i+2^{m+q}}\right) \mathrm{Sq}^{2^{s}}+\tilde{g}_{q}\left(\tilde{\tau}\left(d_{i}\right)\right) \mathrm{Sq}^{2^{s}} \\
& \equiv \tilde{\rho}\left(d_{i+2^{m+q}}\right) \mathrm{Sq}^{2^{s}}+\tilde{g}_{q}\left(\tilde{\tau}\left(d_{i} \mathrm{Sq}^{2^{s}}\right)\right) \\
& =\tilde{\rho}\left(d_{i+2^{m+q}}\right) \mathrm{Sq}^{2^{s}}+\gamma_{i, 2^{s}} \tilde{g}_{q}\left(\tilde{\tau} d_{i-2^{s}}\right) \quad \bmod 2^{k+1} .
\end{aligned}
$$

But the right side of (5.8) is $\tilde{f}_{q}\left(\tilde{\rho}\left(d_{i}\right) \mathrm{Sq}^{2^{s}}\right.$ ), which (using (3.3)(4) since $\mathrm{Sq}^{2^{s}}$ is strict on $i)$ is congruent $\bmod 2^{k+1}$ to $\tilde{f}_{q}\left(\tilde{\rho}\left(d_{i} \mathrm{Sq}^{2^{s}}\right)\right)=\tilde{f}_{q}\left(\tilde{\rho}\left(\gamma_{i, 2^{s}} d_{i-2^{s}}\right)\right)$. Again using the fact that $\mathrm{Sq}^{2^{s}}$ is strict on $i$, so that $m\left(i-2^{s}\right)=m(i)=m$, we may use (5.9) to write the latter as

$$
\left.\gamma_{i, 2^{s}} \tilde{\rho}\left(d_{i-2^{s}+2^{m+q}}\right)+\gamma_{i, 2^{s}} \tilde{g}_{q}\left(\tilde{\tau} d_{i-2^{s}}\right)\right)
$$


Thus the desired congruence holds provided we show that

$$
\tilde{\rho}\left(d_{i+2^{m+q}}\right) \mathrm{Sq}^{2^{s}} \equiv \gamma_{i, 2^{s}} \tilde{\rho}\left(d_{i-2^{s}+2^{m+q}}\right) \bmod 2^{k+1} .
$$

But since $\mathrm{Sq}^{2^{s}}$ is strict on $i$, it is also strict on $i+2^{m+q}$, so again by (3.3)(4)

$$
\tilde{\rho}\left(d_{i+2^{m+q}}\right) \mathrm{Sq}^{2^{s}} \equiv \tilde{\rho}\left(d_{i+2^{m+q}} \mathrm{Sq}^{2^{s}}\right)=\gamma_{i+2^{m+q}, 2^{s}} \tilde{\rho}\left(d_{i-2^{s}+2^{m+q}}\right) \bmod 2^{k+1} .
$$

The only remaining ingredient now is $\gamma_{i+2^{m+q}, 2^{s}} \equiv \gamma_{i, 2^{s}} \bmod 2^{k+1}$. But this follows from the number theoretic Corollary 4.5.

Case II. $\mathrm{Sq}^{2^{s}}$ is final on dimension $i$.

In this case, recall $i-2^{s}=2^{m}-1$.

We begin with the left side of (5.8). By (5.9), $\tilde{f}_{q}\left(\tilde{\rho} d_{i}\right) \mathrm{Sq}^{2^{s}}=\tilde{\rho}\left(d_{i+2^{m+q}}\right) \mathrm{Sq}^{2^{s}}+$ $\tilde{g}_{q}\left(\tilde{\tau} d_{i}\right) \mathrm{Sq}^{2^{s}}$. Now although $\mathrm{Sq}^{2^{s}}$ is final on $i$, it will be strict on $i+2^{m+q}$, so we may apply (3.3)(4), along with (5.10) (which we proved for both Cases I and II), to see that the latter is congruent to

$$
\gamma_{i+2^{m+q}, 2^{s}} \tilde{\rho}\left(d_{i+2^{m+q}-2^{s}}\right)+\tilde{g}_{q}\left(\left(\tilde{\tau} d_{i}\right) \mathrm{Sq}^{2^{s}}\right) \text {. }
$$

However, the second term here is congruent to 0 by $(3.2)(5)$ since $\mathrm{Sq}^{2^{s}}$ is final on $i$.

Moving now to the right side of (5.8), we have

$$
\tilde{f}_{q}\left(\left(\tilde{\rho} d_{i}\right) \mathrm{Sq}^{2^{s}}\right)=\tilde{f}_{q}\left(d_{i} \mathrm{Sq}^{2^{s}}+\left(\tilde{\tau} d_{i}\right) \mathrm{Sq}^{2^{s}}\right) \equiv \gamma_{i, 2^{s}} \tilde{f}_{q}\left(d_{i-2^{s}}\right)+0
$$

again by $(3.2)(5)$. The latter is

$$
\gamma_{i, 2^{s}} \tilde{f}_{q}\left(u_{2^{m}-1}\right)=\gamma_{i, 2^{s}} u_{2^{m}-1+2^{m+q}}=\gamma_{i, 2^{s}} \tilde{\rho}\left(d_{i+2^{m+q}-2^{s}}\right)
$$

since $2^{m}-1+2^{m+q}$ is odd. Now Corollary 4.5 completes the proof of (5.8) since it shows that the Lance coefficients are congruent $\bmod 2^{k+1}$.

This completes the proof that each $f_{q}$ is a fractal $A$-map.

It only remains to show $(1)$, that $f_{q}\left(u_{i}\right)$ is an indecomposable in dimension $i+2^{m(i)+q}$. While it is clear from (5.6) that $f_{q}\left(u_{i}\right)$ is indecomposable, it is far from clear that it is homogeneous of dimension $i+2^{m(i)+q}$. However, from (5.7) and the Witt polynomial form of the terms of (5.9), it is clear this will hold (inductively) provided we show that for $q>1 \tilde{g}_{q}\left(\tilde{\tau} d_{i}\right)$ is homogeneous of dimension $i+2^{m+q}$, where $2^{m} \leq i \leq 2^{m+1}-2$.

We let dim denote the dimension of an element which is homogeneous with respect to dimension, and let $\exp _{x}$ (respectively $\exp _{u}$ ) denote the total exponent of a monomial in the $x$ 's (respectively $u$ 's). Of course dim and exp are both additive on products. Now since $\tilde{g}_{q}\left(x_{2^{m}-1}\right)=u_{2^{m}\left(2^{q-1}+1\right)-1}$, we have

$$
\left(\operatorname{dim}+\exp _{u}\right)\left(\tilde{g}_{q} x_{2^{m}-1}\right)=2^{m}\left(2^{q-1}+1\right)=\left(2^{q-1}+1\right) \cdot\left(\operatorname{dim}+\exp _{x}\right)\left(x_{2^{m}-1}\right) .
$$

Thus

$$
\left(\operatorname{dim}+\exp _{u}\right) \circ \tilde{g}_{q}=\left(2^{q-1}+1\right) \cdot\left(\operatorname{dim}+\exp _{x}\right)
$$

on the generators of $\tilde{L}$, and hence (by additivity on products) on all of $\tilde{L}$. Of course $\operatorname{dim}+\exp _{x}$ is just the weight grading on $\tilde{L}$, and we know from (3.1) that since $\exp _{x}(l)=1, \tilde{\tau} d_{i}$ has weight $i+\left(2^{m+1}-i\right)=2^{m+1}$ (since $i \neq 2^{m}-1$, so 
$\bar{m}(i)=m(i))$. Also, by the definition of $\tilde{g}_{q}$ in (5.5), $\exp _{u} \circ \tilde{g}_{q}=\exp _{x}$ since $q>1$. Using these facts we can solve in (5.12) for

$$
\operatorname{dim}\left(\tilde{g}_{q}\left(\tilde{\tau} d_{i}\right)\right)=\left(2^{q-1}+1\right) \cdot\left(2^{m+1}\right)-\left(2^{m+1}-i\right)=i+2^{m+q}
$$

as claimed.

This completes the proof of Lemma 5.4.

To complete the proof of Theorem 1.2 from Lemma 5.4, merely note $L_{n}$ and $P_{n}$ are $A_{n-1}$ subalgebras of $B_{n}$, and looking at dimensions shows that the tensor product of the inclusions and the $f_{q}$ 's give the desired isomorphism.

We will finish this section by showing that the maps $f_{q}$ have a rather comforting feature. Since our fractal grading using homogeneous generators $u_{i}$ apparently underlies the ability to redimension the fractal $A$-algebra $B_{0}$ with the $f_{q}$ 's, one would hope that $f_{q}$ carries $u_{i}$ to an element which is homogeneous with respect to the grading as well as with respect to dimension.

LEMMA 5.13. $f_{q}\left(u_{i}\right)$ is homogeneous of grade $2^{m(i)+q}$.

PROOF. As with the proof of (1) in Lemma 5.4, this will follow inductively from (5.7) and (5.9) if we show that $\tilde{g}_{q}\left(\tilde{\tau} d_{i}\right)$ has this grade for $q>1$ and $2^{m} \leq i \leq$ $2^{m+1}-2$. Now on the generators $x_{2^{r}-1}$ of $\tilde{L}$,

$$
\operatorname{grade}\left(\tilde{g}_{q}\left(x_{2^{r}-1}\right)\right)=\operatorname{grade}\left(u_{2^{r}-1+2^{r+q-1}}\right)=2^{r+q-1}=2^{q-1} \cdot \operatorname{grade}\left(x_{2^{r}-1}\right)
$$

provided $q>1$, so by additivity grade $\circ \tilde{g}_{q}=2^{q-1} \cdot$ grade on all of $\tilde{L}$. Thus $\operatorname{grade}\left(\tilde{g}_{q}\left(\tilde{\tau} d_{i}\right)\right)=2^{q-1} \cdot \operatorname{grade}\left(\tilde{\tau} d_{i}\right)=2^{q-1} \cdot 2^{m(i)+1}=2^{m(i)+q}$ as claimed.

\section{Proof of Theorem 3.2.}

THEOREM 3.2. Let $\bar{m}=\bar{m}(i)$ as above. Then the homomorphisms $\tau$ and $\tilde{\tau}$ satisfy the following:

(1) $\tilde{\tau}$ extends uniquely to an algebra map $\tilde{B}_{0} \rightarrow \tilde{L}$.

(2) $\tilde{\tau}\left(x_{2^{r}-1}\right)=-x_{2^{r}-1}$ for all $r \geq 1$.

(3) $\tau\left(B_{n}\right) \subset B_{n}$.

(4) $\tilde{\tau}\left(d_{i}\right) \mathrm{Sq}^{2^{s}} \equiv \tilde{\tau}\left(d_{i} \mathrm{Sq}^{2^{s}}\right) \bmod 2^{\nu(i)+1}$ if $\mathrm{Sq}^{2^{s}}$ is strict on i, i.e. if $s<\alpha(i)$ and $2^{m} \leq i-2^{s}<i \leq 2^{m+1}-2$.

(5) $\tilde{\tau}\left(d_{i}\right) \mathrm{Sq}^{2^{s}} \equiv 0 \bmod 2^{\nu(i)+1}$ if $\mathrm{Sq}^{2^{s}}$ is final on $i$, i.e. if $s<\alpha(i)$ and $2^{m}-1=$ $i-2^{s}<i \leq 2^{m+1}-2$.

PROOF. The first step is to show that $\tilde{\tau}$, which was defined only on the integral primitives, actually produces $\tilde{\tau}\left(x_{i}\right)$ with integral coefficients. This will follow from Lemma 2.1 of $[\mathbf{L a}]$, provided we verify the required hypothesis. This reduces to showing that for any odd $j$, if we let

$$
h_{k}\left(x_{1}, \ldots, x_{2^{r}-1}, \ldots\right)=\tilde{\tau}\left(d_{j \cdot 2^{k}}\right)=\left((-l)^{2^{\bar{m}\left(j \cdot 2^{k}\right)+1}-j \cdot 2^{k}}\right)_{\left.\right|_{j \cdot 2^{k}}},
$$

then

$$
h_{k}\left(x_{1}, \ldots, x_{2^{r}-1}, \ldots\right) \equiv h_{k-1}\left(x_{1}^{2}, \ldots, x_{2^{r}-1}^{2}, \ldots\right) \bmod 2^{k} .
$$

Letting $\bar{m}=\bar{m}\left(j \cdot 2^{k}\right)$ and $S l=\sum_{r \geq 1} x_{2^{r}-1}^{2}$, the reader can verify that this means we must show

$$
\left((-l)^{2^{\bar{m}+1}-j \cdot 2^{k}}\right)_{\left.\right|_{j \cdot 2^{k}}} \equiv\left((-S l)^{2^{\bar{m}}-j \cdot 2^{k-1}}\right)_{\left.\right|_{j \cdot 2^{k}}} \bmod 2^{k}
$$


Since $S l \equiv l^{2} \bmod 2$, when we work $\bmod 2^{k}$ we have

$$
(-S l)^{2^{\bar{m}}-j \cdot 2^{k-1}} \equiv\left(-l^{2}\right)^{2^{\bar{m}}-j \cdot 2^{k-1}} \equiv(-1)^{2^{k-1}}(-l)^{2^{\bar{m}+1}-j \cdot 2^{k}} \equiv(-l)^{2^{\bar{m}+1}-j \cdot 2^{k}}
$$

and the result follows.

The second claim of the theorem follows immediately from the definition in (3.1), since $x_{2^{r}-1}=d_{2^{r}-1}$, and $2^{\bar{m}\left(2^{r}-1\right)+1}-\left(2^{r}-1\right)=1$.

To prove the third claim, $\tau\left(B_{n}\right) \subset B_{n}$, it is sufficient to show that $\tau\left(x_{i}\right) \in$ $B_{\alpha(i)-1}$. Looking at the Witt sum, for this it is sufficient by an inductive argument to show that $\tilde{\tau}\left(d_{i}\right) \in \tilde{B}_{\alpha(i)-1} \bmod 2^{\nu(i)+1}$. Looking at the formula for $\tilde{\tau}\left(d_{i}\right)$ we see

$$
\tilde{\tau}\left(d_{i}\right)=(-1)^{i} \sum_{R}\left(\begin{array}{c}
2^{\bar{m}+1}-i \\
r_{1}, r_{2}, \ldots, r_{s}
\end{array}\right) x_{1}^{r_{1}} x_{3}^{r_{2}} \cdots x_{2^{s}-1}^{r_{s}}
$$

where the sum is over all $R=\left(r_{1}, r_{2}, \ldots, r_{s}\right)$ such that $\sum r_{l}\left(2^{l}-1\right)=i$ and $\sum r_{l}=2^{\bar{m}+1}-i$. We will show that if $x_{1}^{r_{1}} \cdots x_{2^{s}-1}^{r_{s}}$ does not lie in $B_{\alpha(i)-1}$ then the coefficient is divisible by $2^{\nu(i)+1}$. Now a monomial $x_{1}^{r_{1}} \cdots x_{2^{s}-1}^{r_{s}}$ lies in $B_{\alpha(i)-1}$ only if all its terms $x_{2^{l}-1}^{r_{l}}$ do, and $x_{2^{l}-1}^{r_{l}}$ is in $B_{\alpha(i)-1}$ provided $\nu\left(r_{l}\right) \geq e\left(\alpha(i)-1,2^{l}-1\right) \geq$ $\alpha(i)-l$. Thus it will suffice to show that

$$
\nu\left(\begin{array}{l}
2^{\bar{m}+1}-i \\
r_{1}, \ldots, r_{s}
\end{array}\right) \geq \nu(i)+1
$$

if $\nu\left(r_{l}\right)<\alpha(i)-l$ for some $l$. This is assured by Lemma 4.6, which was proved in our number theory section.

Now we turn to part (4). Note in this case that $m(i)=\bar{m}(i)=m\left(i-2^{s}\right)=$ $\bar{m}\left(i-2^{s}\right)$, all of which we will call $m$ here. To evaluate the left side we need to compute the action of the Steenrod algebra in $L$. It is convenient to use a different lift of the total Steenrod square Sq to $\tilde{L} \subset \tilde{B}_{0}$. This lift will also obey the integral Cartan formula, and agree with the usual Sq $\bmod 2$, and therefore agree with the Lance lift on $\tilde{\tau}\left(d_{i}\right) \bmod 2^{\nu(i)+1}$. Let $l=\sum_{r \geq 1} x_{2^{r-1}}$ as usual. Now $\bmod 2$,

$$
l \mathrm{Sq}=l+l^{2}+l^{4}+\cdots
$$

so the lift defined by

$$
l \mathrm{Sq}=\sum_{t \geq 0} \frac{1}{2 t+1}\left(\begin{array}{c}
2 t+1 \\
t
\end{array}\right) l^{t+1}
$$

will be correct $\bmod 2$, since $\left(\begin{array}{c}2 t+1 \\ t\end{array}\right)$ is odd precisely if $t+1$ is a power of 2 . In $L$ the action of the Steenrod algebra preserves the weight grading, which is dimension + exponent. Since in this grading $\tilde{\tau}\left(d_{i}\right)$ is homogeneous of grade $2^{m+1}$, we must have $\tilde{\tau}\left(d_{i}\right) \mathrm{Sq}^{2^{s}}$ also homogeneous of grade $2^{m+1} \bmod 2^{\nu(i)+1}$. We are now ready to compute carefully the left side of $(3.2)(4)$, freely using (6.1), Lemma 4.7 , the $Z_{(2)}$ Cartan formula, the fact that $\tilde{\tau} d_{i}$ is a Witt polynomial in $L$, the subscript $\|$ for 
weight projection, | for dimension projection, and the fact that $l$ has exponent 1 :

$$
\begin{aligned}
& \left.\tilde{\tau}\left(d_{i}\right) \mathrm{Sq}^{2^{s}} \equiv(-1)^{i} l^{2^{m+1}-i}\right|_{i} \mathrm{Sq}^{2^{s}} \\
& =(-l)^{2^{m+1}-i} \|_{2^{m+1}} \mathrm{Sq}^{2^{s}}=\left.\left((-l)^{2^{m+1-i}} \|_{2^{m+1}} \mathrm{Sq}\right)\right|_{i-2^{s}} \\
& \left.\quad \equiv\left((-l)^{2^{m+1}-i} \mathrm{Sq} \|_{2^{m+1}}\right)\right|_{i-2^{s}}=\left.\left((-l \mathrm{Sq})^{2^{m+1}-i} \|_{2^{m+1}}\right)\right|_{i-2^{s}} \\
& \left.\quad \equiv\left((-1)^{i} \sum_{t \geq 0}\left(\begin{array}{c}
2 t+2^{m+1}-i \\
t
\end{array}\right) \frac{2^{m+1}-i}{2 t+2^{m+1}-i} l^{t+2^{m+1}-i} \|_{2^{m+1}}\right)\right|_{i-2^{s}} \\
& \quad=\left.(-1)^{i}\left(\begin{array}{c}
2^{s+1}+2^{m+1}-i \\
2^{s}
\end{array}\right) \frac{2^{m+1}-i}{2^{m+1}+2^{s+1}-i} l^{2^{m+1}-\left(i-2^{s}\right)}\right|_{i-2^{s}} \bmod 2^{\nu(i)+1} .
\end{aligned}
$$

The right side of $(3.2)(4)$ is

$$
\tilde{\tau}\left(d_{i} \mathrm{Sq}^{2^{s}}\right)=\gamma_{i, 2^{s}} \tilde{\tau}\left(d_{i-2^{s}}\right)=\left.\frac{i}{i-2^{s}}\left(\begin{array}{c}
i-2^{s} \\
2^{s}
\end{array}\right)(-1)^{i-2^{s}}\left(l^{2^{m+1}-\left(i-2^{s}\right)}\right)\right|_{i-2^{s}} .
$$

Note that here the hypothesis $2^{m} \leq i-2^{s}$ is crucial to the validity of this formula for $\tilde{\tau}\left(d_{i-2^{s}}\right)$. Thus to complete the proof we need to show that

$$
\begin{aligned}
(-1)^{i} & \left(\begin{array}{c}
2^{s+1}+2^{m+1}-i \\
2^{s}
\end{array}\right) \frac{2^{m+1}-i}{2^{m+1}+2^{s+1}-i} \\
& \equiv(-1)^{i-2^{s}} \frac{i}{i-2^{s}}\left(\begin{array}{c}
i-2^{s} \\
2^{s}
\end{array}\right) \bmod 2^{\nu(i)+1} .
\end{aligned}
$$

Corollary 4.3 guarantees the validity of this final congruence, completing the proof of part (4).

Finally, we prove part (5). Since all of $A$ preserves the weight grading mod 2 on $\tilde{L}$, Lance's squares must preserve it $\bmod 2^{\nu(i)+1}$ on $\tilde{\tau} d_{i}$. Now $\tilde{\tau} d_{i}$ has weight $2^{m+1}$, so the element $\left(\tilde{\tau} d_{i}\right) \mathrm{Sq}^{2^{s}}$ has weight $2^{m+1} \bmod 2^{\nu(i)+1}$. But since $\mathrm{Sq}^{2^{s}}$ is final on $i$, it also lies in dimension $2^{m}-1$, and we claim no nonzero element in this dimension can have weight that large. This is because in terms of the $x_{2^{r}-1}$ 's, weight $=$ dimension + exponent on $\tilde{L}$, and exponent $\leq$ dimension, so weight $\leq$ $2 \cdot$ dimension.

\section{REFERENCES}

[Ba] A. Baker, Husemoller-Witt decompositions and actions of the Steenrod algebra, preprint.

[BM] A. P. Bahri and M. E. Mahowald, A direct summand in $H^{*}\left(M O\langle 8\rangle ; Z_{2}\right)$, Proc. Amer. Math. Soc. 78 (1980), 295-298.

[BP] E. H. Brown and F. P. Peterson, On the stable decomposition of $\Omega^{2} S^{r+2}$, Trans. Amer. Math. Soc. 243 (1978), 287--298.

[Br] R. A. Brualdi, Introductory combinatorics, Elsevier, North-Holland, New York, 1977.

[D1] D. M. Davis, On the cohomology of $M O\langle 8\rangle$, Sympos. on Algebraic Topology in Honor of Jose Adem (Samuel Gitler, ed.), Contemp. Math., vol. 12, Amer. Math. Soc., Providence, R. I., 1982, pp. 91-104.

[D2] _ The splitting of $B O\langle 8\rangle \wedge$ bo and $M O\langle 8\rangle \wedge b o$, Trans. Amer. Math. Soc. 276 (1983), 671-683.

[D3] _ Connective coverings of BO and immersions of projective spaces, Pacific J. Math. 76 (1978), 33-42.

[DGIM] D. M. Davis, S. Gitler, W. Iberkleid, and M. Mahowald, The orientability of vector bundles with respect to certain spectra, Bol. Soc. Mat. Mex. (2) 4 (1979), 49-55. 
[DM1] D. M. Davis and M. Mahowald, The nonrealizability of the quotient $A / / A_{2}$ of the Steenrod algebra, Amer. J. Math. 104 (1982), 1211-1216.

[DM2] _ Ext over the subalgebra $A_{2}$ of the Steenrod algebra for stunted projective spaces, Proc. Conf., Current Trends in Algebraic Topology (Univ. of Western Ontario, 1981), Canad. Math. Soc. Conf. Proc., vol. 2, Part 1, 1982, pp. 297-342.

[DM3] - The immersion conjecture for $R P^{8 k+7}$ is false, Trans. Amer. Math. Soc. 236 (1978), 361-383.

[GMP] V. Giambalvo, R. Mines, and D. J. Pengelley, Two-adic congruences between binomial coefficients, preprint.

[GP] V. Giambalvo and D. J. Pengelley, The homology of MSpin, Math. Proc. Cambridge Philos. Soc. 95 (1984), 427-436.

[GPR] V. Giambalvo, D. J. Pengelley and D. C. Ravenel, Fractal structures in $H_{*} B O$ and their applications to cobordism, Proc. Lefschetz Centennial Conf. (Mexico City, 1984), Contemp. Math., vol. 58, Part II, Amer. Math. Soc., Providence, R. I.,1987, pp. 43-50.

[Hu] D. Husemoller, The structure of the Hopf algebra $H_{*}(B U)$ over a $Z_{(p)}$-algebra, Amer. J. Math. 93 (1971), 329-349.

[K1] S. O. Kochman, An algebraic filtration of $H_{*}\left(M O ; Z_{2}\right)$, Proc. Edinburgh Math. Soc. (2) 26 (1983), 313-317.

[K2] _ An algebraic filtration of $H_{*} B O^{\prime}$, Proc. Conf. on Homotopy Theory (Northwestern Univ., Evanston, Ill., 1982), Contemp. Math., vol. 19, Amer. Math. Soc., Providence, R. I., 1983, pp. 115-143.

[La] T. Lance, Steenrod and Dyer-Lashof operations on BU, Trans. Amer. Math. Soc. 276 (1983), 497-510.

[Le] A. M. Legendre, Théorie des nombres, in Vol. 1, 3rd ed., Didot Freres, Paris, 1830.

[Li] A. Liulevicius, Characteristic numbers, (Proc. Conf., Memorial Univ., Newfoundland, St. John's, 1973) (S. Thomeier, ed.) Topology and Its Applications, Lecture Notes in Pure and Appl. Math., vol. 12, Dekker, New York, 1975, pp. 1-25.

[M] B. Mandelbrot, personal communication.

[P1] D. J. Pengelley, The A-algebra structure of Thom spectra: $M S O$ as an example, Proc. Conf. Current Trends in Algebraic Topology (Univ. of Western Ontario, 1981), Canad. Math. Soc. Conf. Proc., vol. 2, Part 1, 1982, pp. 511-513.

[P2],$H^{*}(M O\langle 8\rangle ; Z / 2)$ is an extended $A_{2}^{*}$-coalgebra, Proc. Amer. Math. Soc. 87 (1983), 355356.

[PR] H. O. Peitgen and P. H. Richter, Schönheit im chaos (Frontiers of chaos), Forschungsgruppe Complexe Dynamik, Universität Bremen, 1985.

[Si] D. Singmaster, Divisibility of binomial and multinomial coefficients by primes and prime powers, A Collection of Manuscripts Related to the Fibonacci Sequence, 18th Anniversary Volume (V. Hoggatt, Jr., and M. Bicknell-Johnson, eds.), The Fibonacci Association, Santa Clara, Calif., 1980, pp. 98-113.

[Sn] V. P. Snaith, A stable decomposition of $\Omega^{n} S^{n} X$, J. London Math. Soc. (2) 7 (1974), 577-583.

[St] R. E. Stong, Determination of $H^{*}\left(B O(k, \ldots, \infty), Z_{2}\right)$ and $H^{*}\left(B U(k, \ldots, \infty), Z_{2}\right)$, Trans. Amer. Math. Soc. 107 (1963), 526-544.

[Sw] R. M. Switzer, Algebraic topology homotopy and homology, Grundlehren Math. Wiss. 212, Springer, New York, 1975.

Department of Mathematics, University of Connecticut, Storrs, ConnecTICUT 06268

Department of Mathematics, New Mexico State University, Las Cruces, NEW MEXICO 88003

Department of Mathematics, University of Washington, Seattle, WashingTON 98195 